\title{
Quantum Spacetime: a Disambiguation ${ }^{\star}$
}

Gherardo PIACITELLI

SISSA, Via Bonomea 265, 34136, Trieste, Italy

E-mail: gherardo@piacitelli.org

Received April 29, 2010, in final form September 15, 2010; Published online September 23, 2010

doi:10.3842/SIGMA.2010.073

\begin{abstract}
We review an approach to non-commutative geometry, where models are constructed by quantisation of the coordinates. In particular we focus on the full DFR model and its irreducible components; the (arbitrary) restriction to a particular irreducible component is often referred to as the "canonical quantum spacetime". The aim is to distinguish and compare the approaches under various points of view, including motivations, prescriptions for quantisation, the choice of mathematical objects and concepts, approaches to dynamics and to covariance.
\end{abstract}

Key words: quantum spacetime; covariance; noncommutative geometry; doubly special relativity

2010 Mathematics Subject Classification: 46L65; 81T75; 83C65; 58B32

\section{Contents}

1 Introduction $\quad 2$

2 Motivations $\quad 3$

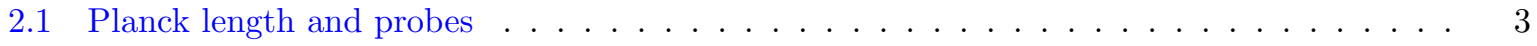

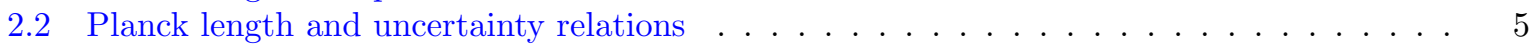

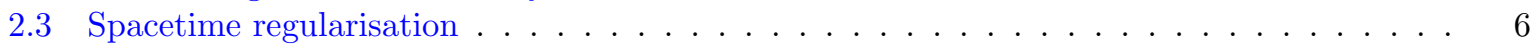

2.4 The meaning of "minimal length", and Doubly Special Relativity . . . . . . . . . . . . 6

$\begin{array}{lll}3 & \text { Which algebra? } & \mathbf{7}\end{array}$

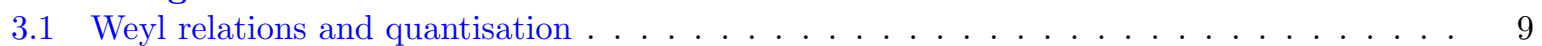

3.2 Moyal expansion . . . . . . . . . . . . . . . . . . . . . . . . . . 10

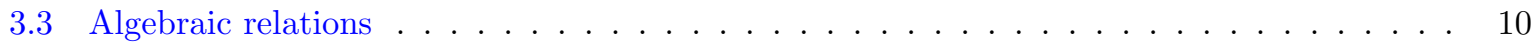

3.4 Dangers of the Moyal expansion . . . . . . . . . . . . . . . . . . . . 11

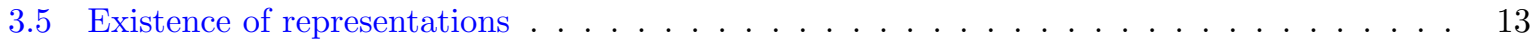

4 Covariance and the DFR model $\quad 14$

4.1 "Be wise, covariantise" . . . . . . . . . . . . . . . . . . . . . . . . . . . . 14

4.2 Representations . . . . . . . . . . . . . . . . . . . . . 16

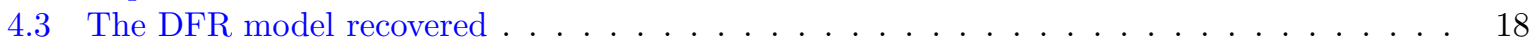

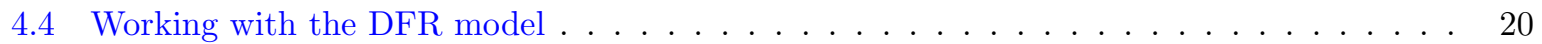

4.5 Classical limit . . . . . . . . . . . . . . . . . . . . . . . . . . 22

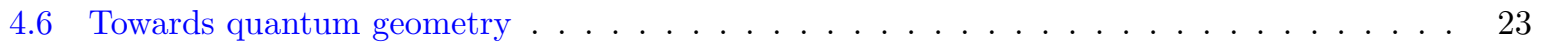

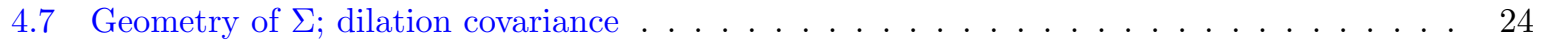

5 The "canonical" quantum spacetime $\quad 25$

5.1 Time/space commutative models and representations . . . . . . . . . . . . . 25

5.2 "Canonical" quantum spacetime and DFR model . . . . . . . . . . . . . . . . . 27

5.3 "Canonical quantum spacetime" and localisation states . . . . . . . . . . . . . 28

5.4 Twisted covariance . . . . . . . . . . . . . . . . . . . . . . . . . . 29

*This paper is a contribution to the Special Issue "Noncommutative Spaces and Fields". The full collection is available at http://www.emis.de/journals/SIGMA/noncommutative.html 
6 Quantum field theory 33

6.1 Local quantum fields . . . . . . . . . . . . . . . . . . . . . . . . 33

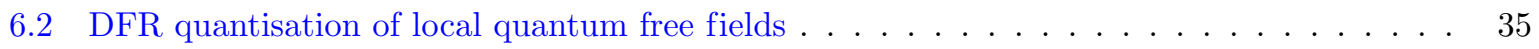

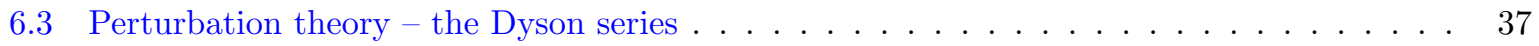

6.4 Formal unitarity is not violated! . . . . . . . . . . . . . . . . . . . . 39

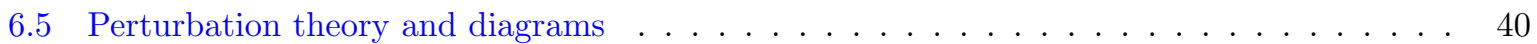

6.6 Euclidean methods and UV/IR mixing . . . . . . . . . . . . . . . . . . . 40

References

42

\section{Introduction}

Within the class of models of quantum spacetime which are defined by imposing non trivial commutation relations on the coordinates, there are quite different approaches on the market. A list of differences should include at least

a) different aims and motivations,

b) different philosophies in the quantisation prescriptions,

c) different classes of algebras (as mathematical objects),

d) different approaches to dynamics and,

e) last but not least, different approaches to covariance.

Of course the above differences are not all independent.

In this review, some comments on the above issues will be made under a narrow perspective. I will not attempt a thorough description of the immense literature, both for reasons of space and lack of knowledge; I apologise in advance for any unforgivable omission. Very little of the material and the comments presented is new, although some remarks only previously arose in discussions.

The root of motivations for these investigations is shared by everybody in this field: the hope that a full theory of quantum gravity could possibly emerge from completing the transition to a full quantum "description of nature", encompassing geometry as well. Another common key remark is that the ultraviolet nightmare which plagues perturbative quantum field theory might be a symptom that geometry is not classical in the small. Moreover, by a consistence argument, sharp localisation should not be expected to be possible at all scales: indeed, at very small scales it should lead to some instability of the geometric background, as localisation in sharp regions might induce the formation of closed horizons. This comment is very old and took many shapes; depending on the chosen shape, it leads to different approaches to the problem; we will discuss it in Section 2. There, we also will shortly comment on Doubly Special Relativity, and show that singly Special Relativity is already multiply special, at least in some models.

On a more detailed ground, the above comments have been interpreted in at least three different contexts: string theory, quantum field theory, and quantum mechanics.

We will not discuss the string-theoretical aspects because I feel incompetent.

In quantum field theory, the quantisation of the localisation algebra should be interpreted as the framework providing a non-commutative replacement for pointwise products of local free fields in interaction terms; we will discuss this in Section 6.

The quantum mechanical approach, instead, involves a change in the commutation relations for the momenta as well: while in a non-commutative algebra (with translation covariance) momenta pairwise commute and generate the usual action of the classical translation group, 
in this approach ${ }^{1}$ momenta and coordinates with mixed commutation relations should ideally replace the usual Schrödinger operators at small scales. We will not discuss this approach; indeed, because of the extremely high energies expected to occur in processes at Planck scale, a Planck scale modification of quantum mechanics does not seem to be a physically interesting limit.

The choice of the relevant algebraic structure and quantisation prescription is somewhat critical, and is full of consequences on the technical and conceptual ground as well. The issue is not at all a marginal technical one. For example, it is often said that to a great extent quantum physics is a spectral theory. But if the relations

$$
\left[\boldsymbol{x}^{\mu}, \boldsymbol{x}^{\nu}\right]=i \theta^{\mu \nu} \boldsymbol{I}
$$

instead of being understood as regular relations among selfadjoint operators on some Hilbert space, are taken as the defining relations between a finite set of Hermitean generators of an abstract *-algebra (which turns out not $C^{*}$ ), then every non trivial element in that algebra (including the selfadjoint coordinates $\boldsymbol{x}^{\mu}$ ) has the whole complex plane as its spectrum, and the spectral theory is completely trivial. Moreover, the transition from the above relations to the apparently equivalent notation

$$
\boldsymbol{x}^{\mu} \star \boldsymbol{x}^{\nu}-\boldsymbol{x}^{\nu} \star \boldsymbol{x}^{\mu}=i \theta^{\mu \nu}
$$

is far from innocent: if $\star$ is understood as a twisted product of symbols, the above cannot coexist with the so called Weyl quantisation: they are mutually exclusive. This will be discussed in Section 3.

The importance of identifying the representations of the relations is made even more evident when we consider for example time/space commutativity; we will see in Section 5.1 that there is a variety of situations, including the possibility that no representation exists.

The full DFR model is described in some detail in Section 4, where an attempt is made to make some concepts more accessible, notwithstanding the technical implications. The material is organised so to easily discuss related concepts available in the literature.

In Section 5 we comment on some flavours of the "canonical" quantum spacetime. In particular the relations with twisted covariance are discussed in Section 5.4.

Section 6 is devoted to quantum field theory (QFT) on quantum spacetime (QST). We will recall the approach based on the Gell-Mann-Low formula with a non local interaction, and focus on the issue of time-ordering, and its relations with unitarity. We also shortly recall some basic facts about the diagrams, with a disambiguation between the Filk rules and those arising from the Dyson series. We finally will shortly comment on the availability of Euclidean methods, and on the IR/UV mixing.

We will not conclude with an outlook, for which I refer instead to [1, 2].

\section{Motivations}

\subsection{Planck length and probes}

If spacetime is classical (e.g. the Minkowski spacetime), the non trivial Heisenberg space/momentum uncertainty relations

$$
\Delta x_{j} \Delta p_{j} \geqslant \frac{\hbar}{2}
$$

\footnotetext{
${ }^{1}$ These approaches are usually referred to as "non-commutative quantum field theory (NQFT)" and "noncommutative quantum mechanics (NCQM)". However, since by its very nature quantum physics is non commutative, "non-commutative quantum" contrasted with "quantum" alone is really an awful terminology, in my humble opinion. Since words do matter, we shall dismiss it here.
} 
should be ideally complemented with the missing $4^{\text {th }}$ component time/energy uncertainty relation

$$
\Delta t \Delta E \geqslant \frac{\hbar}{2}
$$

(all other uncertainty relations being trivial). Indeed in classical Hamiltonian mechanics, time and energy are canonically conjugate variables.

Although there are no time and position observables in a relativistic theory, the above relations can be taken as an indication that the localisation process implies an energy transfer to spacetime. The higher is the precision of localisation in time (small $\Delta t$ ), the higher is the energy transferred to the geometric background; if localisation is also confined

in a small space volume,

then the energy density induced by localisation might produce a closed horizon (a black hole). This would lead to a paradoxical situation, where the horizon would trap any information, and we would face a localisation process with no output. Thus, in order to have a consistent operational description of spacetime, geometry should be modified in the small so to prevent "too sharp" localisation.

This remark is very old and it is probably fair to ascribe it to folklore. As far as I know, the first who tried to give it a quantitative content was Mead in $[3]^{2}$. From a quite general argument based on the "Heisenberg microscope", he deduced that, to avoid the above mentioned spacetime instability, the size $\Delta r$ of probes should be bounded below by the Planck length

$$
\lambda_{P}=\left(\frac{G \hbar}{c^{3}}\right)^{1 / 2} \simeq 1.6 \times 10^{-33} \mathrm{~cm} .
$$

Reduced to its essence, Mead's argument is based on the remark that if one considers a mass $m$ with its associated Compton wavelength $\lambda(m)$ and Schwarzschild radius $R(m)$, the condition $\lambda(m) \sim R(m)$ is fulfilled for $m \sim m_{P}$, the Planck mass, and we find $\lambda\left(m_{P}\right) \sim R\left(m_{P}\right) \sim \lambda_{P}$.

Mead deduced from this the necessity of a minimal uncertainty of order of $\lambda_{P}$. While this statement is somewhat questionable, certainly the basic argument provides a good motivation for assuming that the Planck length is the relevant scale where gravitation and quantum physics should meet.

Analogous conclusions were drawn by Amati, Ciafaloni and Veneziano, from a different perspective [5] and in the context of string theory; they found a relation of the form

$$
\Delta r \geqslant \frac{\hbar}{\Delta p}+\alpha^{\prime} \Delta p
$$

where $\alpha^{\prime}$ is a positive constant depending on Regge's slope and the gravitation constant. For positive $x$ 's, $\hbar / x+\alpha^{\prime} x$ takes its minimum value $\lambda=2 \sqrt{\alpha^{\prime} \hbar}$ at $x=\sqrt{\hbar / \alpha^{\prime}}$. Hence they found again the lower estimate

$$
\Delta r \geqslant \lambda_{P} \sim 10^{-33} \mathrm{~cm}
$$

for the size of an admissible probe.

The same relation (2.2) and the same conclusions were reobtained by Maggiore [6], with a "model independent" argument quite close to that of Mead; probably Maggiore was not aware of Mead's analysis.

\footnotetext{
${ }^{2}$ Actually that paper was submitted in 1961, but underwent referee troubles; see the interesting letter of Mead to Physics Today [4].
} 


\subsection{Planck length and uncertainty relations}

The argument of Mead and Maggiore is indeed model independent from the dynamical point of view, but there is a hidden assumption [2], which also is present in [5]. Namely that the uncertainties of the three space coordinates must be of the same order. Indeed, to derive (2.2) the interaction of probes with spacetime is explicitly modeled (at least by Mead and Maggiore) by means of a solution of the Einstein equations with spherical symmetry: the " $\Delta(p)$ " showing up in (2.2) is the total black hole momentum. Hence the only conclusion which is obtained in those references is that

If we assume that spacetime only can be probed with spherically symmetric devices, than there must be a minimal uncertainty.

But why should probes be spherical? Let us come back to the heuristic discussion at the beginning of the section. All the point is hidden in (2.1). Indeed, if we assume that the localisation region has spherical symmetry, then all the $\Delta x_{j}$ 's are equal to each other, and in particular they all must be small in order to obtain a small volume. But a small volume can also be obtained with some large $\Delta x_{j}$; it only is necessary that $\prod_{j} \Delta x_{j}$ is small. Hence, if we dismiss the assumption of spherically symmetric probes, the argument of Mead for the necessity of a minimal uncertainty for a length measurement is not compelling any more ${ }^{3}$. Indeed, this remark was the starting point of the investigation of [7].

It is interesting to note that Maggiore was able to rederive the relation (2.2) from suitably modified commutation relations between positions and momenta [8]. He found that the so called $\kappa$-Poincaré commutation relations induce uncertainty relations such that

in a certain regime

the relation (2.2) is fulfilled. However, the close (2.3) implies that those relations are not always valid; they only are asymptotically valid under limiting conditions which imply spherical symmetry. Maggiore then concluded, once again, that there must be a minimal uncertainty; but if one looks instead at the unrestricted uncertainty relations, no absolute bounds can be deduced for the values of a single coordinate.

Starting from a quantitative heuristic analysis (using quantum field theory) of the initial remark of this section, Doplicher, Fredenhagen and Roberts wrote down operationally motivated uncertainty relations:

$$
\begin{aligned}
& \Delta x^{0}\left(\Delta x^{1}+\Delta x^{2}+\Delta x^{3}\right) \geqslant \lambda_{P}^{2}, \\
& \Delta x^{1} \Delta x^{2}+\Delta x^{1} \Delta x^{3}+\Delta x^{2} \Delta x^{3} \geqslant \lambda_{P}^{2} .
\end{aligned}
$$

Moreover, they found commutation relations inducing those uncertainty relations [7]. This led them to formulate their model (DFR model); we shall discuss those relations in some detail, later in this review. Let us anticipate however that no minimal length for a length measurement shows up in that model: coordinates have purely continuous spectrum and nothing prevents sharp localisation in some of the coordinates; it is only the precision in the simultaneous localisation of all the coordinates which is bounded by uncertainty relations involving the Planck length as a characteristic length of the model.

\footnotetext{
${ }^{3}$ The situation is much alike that of usual quantum mechanics (for a particle on the line, for simplicity). There the Heisenberg uncertainty relations $\Delta \boldsymbol{P} \Delta \boldsymbol{Q} \geqslant \hbar / 2$ do not imply that $\boldsymbol{P}, \boldsymbol{Q}$ have discrete spectrum. On the contrary, it is well known that the Schrödinger operators have purely continuous spectrum. The rectangular Planck cells used in quantum statistical mechanics have no fixed shape; for all computations it is sufficient to know their area $\hbar / 2$.
} 


\subsection{Spacetime regularisation}

We may observe, as an additional motivation for spacetime quantisation, that relativistic quantum field theory has defeated for almost a century every attempt to construct a non perturbative model in physical (i.e. 4) dimensions: only free fields are known as exact models. Moreover, the perturbative approach is plagued by severe divergences, called "ultraviolet" because they involve the behaviour at small distances. Notwithstanding the general success of renormalisation theory ${ }^{4}$ and the wonderful experimental validation of lower order renormalised contributions, no exact perturbative limit theory is known in $d=4$, which is not trivial (i.e. not free). It is rather reasonable to conclude that some internal contradiction in the theory arises when we push locality to the "infinitely small scale". Hence an additional motivation for spacetime quantisation is the hope for obtaining well defined interacting models, as a byproduct of the regularising effect of the quantum texture of spacetime.

For the sake of completeness, we mention that the first model of quantum spacetime based on non-commuting coordinates is due to Snyder [9]. This model was motivated precisely as an attempt to mimic lattice regularisation of the perturbative terms in a covariant way. The elegant idea was abandoned when the renormalisation program gave an effective solution to the perturbative problem. Although Snyder was aware of the conceptual implications of spacetime quantisation and of the possible follow-up of his research, he did not develop this line. Indeed, from his point of view uncertainty relations were a possible source of nuisance, and he only computed them to check that they would have not spoiled the consistency of the picture underlying his framework (see especially the very last sentence of his paper).

\subsection{The meaning of "minimal length", and Doubly Special Relativity}

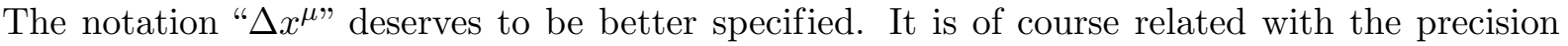
with which the position $x^{\mu}$ can be known. It is however necessary to distinguish the concepts of minimal length and minimal uncertainty.

If the position is described by a selfadjoint operator $\boldsymbol{x}^{\mu}$ under a quantum perspective, then it is understood that the uncertainty of the position in a state $\omega$ is given by

$$
\Delta x^{\mu}=\Delta_{\omega}\left(\boldsymbol{x}^{\mu}\right)=\sqrt{\omega\left(\left(\boldsymbol{x}^{\mu}-\omega\left(\boldsymbol{x}^{\mu}\right) \boldsymbol{I}\right)^{2}\right)}=\sqrt{\omega\left(\boldsymbol{x}^{\mu 2}\right)-\omega\left(\boldsymbol{x}^{\mu}\right)^{2}} .
$$

It is clear that, for any spectral value $a$ of $\boldsymbol{x}^{\mu}$, there is a state $\omega$ such that $\omega\left(\boldsymbol{x}^{\mu}\right)=a$ with arbitrarily small uncertainty ${ }^{5}$, whatever commutation relations the coordinates do fulfil. Hence an absolute lower bound for the uncertainty of one coordinate is incompatible with the possibility of describing the coordinates by means of operators.

For example, since Snyder's model is defined in terms of selfadjoint coordinates, it is always possible to sharply localise in one Snyder coordinate alone. There, however, interest is appointed to the fact that the spectrum of each coordinate $\boldsymbol{x}^{\mu}$ is of the form $\lambda \mathbb{Z}$ for some parameter $\lambda$. In this model we may speak of a minimal length, defined as the minimal separation $\Delta x^{\mu}$ between two possible values of the same coordinate (which plays the rôle of the precision of an instrument, instead of its error); but this now means to attach a different meaning to the notation $\Delta x^{\mu}$. Anyhow, we see here a first example where the concept of minimal length has a meaning even when all the coordinates can be separately sharply localised.

In general, relative bounds on the precision of joint localisation in two or more non commuting coordinates arise, as a consequence of the commutation rules, by (variants of) the generalised

\footnotetext{
${ }^{4} \mathrm{~A}$ rather complicate recursive local covariant strategy for removing divergences from the perturbative series.

${ }^{5}$ If $a$ is an eigenvalue with eigenvector $|a\rangle$, take $\omega(a)=\langle a|\cdot| a\rangle /\langle a \mid a\rangle$; if $a$ is in the continuous spectrum, replace the generalised eigenket $|a\rangle$ with a sufficiently good finite length approximation.
} 
Heisenberg uncertainty theorem ${ }^{6}$. In the case of Snyder's model, such uncertainty relations are not known (Snyder never published the computations he made). In the case of the DFR model such relations are known, and actually were the motivations for the investigation.

In the DFR model, though the coordinates are selfadjoint and have spectrum $\mathbb{R}$, there also is room for a minimal length. Indeed the "Euclidean square distance" ${ }^{7}$ operator is bounded below by $2 \lambda_{P}^{2}$. Note that the Euclidean length is not a Lorentz invariant, so that inequivalent observers may fail to agree on states achieving the minimum. However, the minimal length (in the sense above) is well defined and has the same value for every observer, and is thus a general property of the model.

Above, we speak of Euclidean distance of a quantum event from a classical point, which has no physical interpretation. However there is a more refined version of this comment, which shows that there also is a minimal Euclidean distance between two independent quantum events [10].

It is often stated that the existence of a minimal ${ }^{8}$ length is incompatible with ordinary Lorentz covariance, because of Lorentz-Fitzgerald contraction; and that, as a consequence, it is necessary to "deform" covariance in some appropriate sense (e.g. in the sense of Quantum Groups). This in turn is interpreted in the general phenomenological framework of "Doubly Special Relativity" (DSR). We will not enter here in a description of DSR (see e.g. [11]). We only observe that the DFR analysis provides a well defined model where

- the coordinates are described by non commuting coordinates, which are selfadjoint operators on some Hilbert space,

- there is a continuous unitary representation of the (usual, undeformed) Poincaré group on the same Hilbert space,

- the above representation implements ordinary covariance of the coordinates (quantum symmetry in the usual sense of Wigner), and

- there is a minimal length which is the same for all equivalent observers.

Hence Lorentz-Fitzgerald contraction is not at all incompatible with the presence of a minimal length; the quest for DSR does not forces us in principle to deform covariance, though of course deformed covariance might well be anyway interesting for other reasons.

In a sense, usual special relativity is already multiply special. To encompass $n$-ply special relativity it is sufficient to find non commutative algebras with $n$ universal parameters, equipped with an undeformed action of the usual Poincaré group by automorphisms; there is plenty of such examples (see e.g. [12]).

\section{Which algebra?}

In this section we will begin our disambiguation by discussing and comparing some necessary mathematical tools and concepts. In particular we will show that defining the algebra of quantum spacetime through Weyl quantisation, or as an algebra generated by finitely many elements and relations, leads to profoundly different mathematical structures, and we will shortly discuss the consequences of this.

Let us keep apart for the moment the issue of covariance, and consider relations of the form

$$
\left[\boldsymbol{x}^{\mu}, \boldsymbol{x}^{\nu}\right]=i \theta^{\mu \nu} \boldsymbol{I},
$$

where $\theta^{\mu \nu}$ is a non zero antisymmetric matrix with real entries. Assume moreover that the $\boldsymbol{x}^{\mu}$ are selfadjoint.

\footnotetext{
${ }^{6}$ For any $\boldsymbol{A}=\boldsymbol{A}^{*}, \boldsymbol{B}=\boldsymbol{B}^{*}$ and state $\omega, \Delta_{\omega}(\boldsymbol{A}) \Delta_{\omega}(\boldsymbol{B}) \geqslant \frac{1}{2}|\omega([\boldsymbol{A}, \boldsymbol{B}])|$.

${ }^{7}$ From the (classical) origin; but note that the model has translation covariance.

${ }^{8}$ The "smallness" of this length is not relevant here; we'd better call it a universal length.
} 
Let us show that a $*$-algebra $\mathcal{A}_{\theta}$ with unit, which contains selfadjoint elements $\boldsymbol{x}^{\mu}$ fulfilling the above relations, cannot be faithfully represented as an algebra of bounded operators.

To see this ${ }^{9}$, let $\bar{\mu}, \bar{\nu}$ be some fixed choice of the values of the dummy indices $\mu, \nu$, such that $\theta^{\bar{\mu}} \bar{\nu} \neq 0$. If we rename $\boldsymbol{P}=\boldsymbol{x}^{\bar{\nu}}, \boldsymbol{Q}=\boldsymbol{x}^{\bar{\mu}}, \hbar=\theta^{\bar{\mu} \bar{\nu}}$, we discover the well known Canonical Commutation Relation (CCR)

$$
[\boldsymbol{P}, \boldsymbol{Q}]=-i \hbar \boldsymbol{I}
$$

hidden in the spacetime commutation relations (as a purely mathematical fact, deprived of any physical interpretation). It is well known from elementary quantum mechanics that the CCR cannot be represented by means of bounded operators: at least one among position and momentum must be unbounded. It follows that the algebra $\mathcal{A}_{\theta}$ cannot have a faithful $*$-representation by bounded operators on some Hilbert space (for, otherwise, there also would be a bounded representation of the CCR). This applies in particular if $\mathcal{A}_{\theta}$ is precisely the algebra generated by the relations (3.1), as we shall assume from now on.

In other words, $\mathcal{A}_{\theta}$ has no $C^{*}$-completions at all (technically: there is no pre- $C^{*}$-norm on $\mathcal{A}_{\theta}$ ).

Existence of $C^{*}$-completions is not a marginal technical detail for quantum physics. In a generic $*$-algebra, all one can say about the spectrum of a selfadjoint element is that it is invariant under complex conjugation; but it might well fail to be contained by the real axis. Moreover, "functions" of elements in a generic *-algebra (even a Banach one) may be defined to a limited extent. The $C^{*}$ property ${ }^{10}$ is the minimal requirement ensuring that spectra of selfadjoints are real, and that there is a useful notion of functional calculus, mapping the spectra in the natural way. These two properties, in turn, are indispensable for a sound physical interpretation.

The existence of sufficiently many representations of (regular) commutation relations is a conditio sine qua non for the existence of $C^{*}$-completions; whence comes the quantum motto

\section{"No DEFORMATION Without REPRESENTATION!"}

A completely different approach to the construction of the spacetime algebra was proposed much earlier in [7], in a more general setting. The idea is to follow the approach proposed by Weyl (and adopted by von Neumann) in the case of quantum mechanics, where a canonical map is defined, which sends functions into operators, and is called Weyl quantisation; the functions in the domain of the quantisation are called "symbols". By pulling back the operator product to symbols, one obtains the $*$-algebra $\mathcal{E}_{\theta}$ of "symbols", equipped with a non-commutative product $\star$ which is a deformation of the usual pointwise product (e.g. in the sense of [14]); we will describe it in more detail here below. It was shown in [7] that, for a large class of matrices $\theta$, this $*$-algebra may be faithfully represented as a $*$-algebra of bounded operators on some Hilbert space $(=$ it has a unique $C^{*}$-completion). Note however that, though this $*$-algebra is indirectly defined by commutation relations among the coordinates, it does not contain the coordinates themselves! The quantum coordinates $\boldsymbol{x}^{\mu}$ arise as the unbounded selfadjoint operators on the Hilbert space which are canonically associated with the Weyl quantisation.

Hence, the $*$-algebras $\mathcal{A}_{\theta}, \mathcal{E}_{\theta}$ are non-isomorphic in a very substantial way, which can not be circumvented, and which leads to very different frameworks, both on the conceptual and technical side.

As a remarkable example, note that in the algebra $\mathcal{A}_{\theta}$ the Weyl quantisation is not available, since there is no such object in $\mathcal{A}_{\theta}$ as $e^{i k_{\mu} \boldsymbol{x}^{\mu}}$; nor there is any natural notion of completion or multipliers algebra of $\mathcal{A}_{\theta}$, where to give a meaning to the defining series of $e^{i k_{\mu} \boldsymbol{x}^{\mu}}$. Even worse is that the spectral theory is completely trivial in $\mathcal{A}_{\theta}$, as we shall soon see.

\footnotetext{
${ }^{9}$ For a nice purely $C^{*}$-algebraic argument not relying on von Neumann uniqueness, see [13, $\left.\S 11\right]$

${ }^{10} \mathrm{~A} C^{*}$-algebra is a Banach $*$-algebra enjoying $\left\|a^{*} a\right\|=\|a\|^{2}$ for any of its elements $a$. A $C^{*}$-algebra admits a unique $C^{*}$-norm, fully determined by the algebraic structure.
} 
In the remaining of this section we will describe more precisely the way in which the algebras $\mathcal{E}_{\theta}$ and $\mathcal{A}_{\theta}$ are constructed, and we will make some of the above remarks more precise. We also will comment in some detail on the most dangerous cowboy downtown, the Moyal expansion. Since in the literature the same words and symbols are used in the two different contexts for related concepts, we will be a little pedantic, in order to detect the sources of ambiguity. Although references will be made to advanced mathematical concepts at some points, hopefully the bulk material should appear reasonably accessible.

\subsection{Weyl relations and quantisation}

By the BCH formula, (3.1) formally implies the relations

$$
e^{i k_{\mu} \boldsymbol{x}^{\mu}} e^{i h_{\nu} \boldsymbol{x}^{\nu}}=e^{-\frac{i}{2} h_{\mu} \theta^{\mu \nu} k_{\nu}} e^{i(h+k)_{\mu} \boldsymbol{x}^{\mu}} .
$$

Contrary to (3.1), (3.2) is completely unambiguous if the $\boldsymbol{x}^{\mu}$ are understood to be selfadjoint operators.

By a simple argument ([7]; see also Section 3.5) it is possible to show that, for a large class of matrices $\theta$, there exist unbounded selfadjoint operators $\boldsymbol{x}^{\mu}$ on a Hilbert space $\mathfrak{H}$, fulfilling the above relations as relations between unitary operators. For a smaller, yet still large class of matrices, the operators $\boldsymbol{x}^{\mu}$ are also unique (up to multiplicity and equivalence); we assume this to be the case.

In [7] it also was proposed to adapt the Weyl quantisation prescription

$$
f(\boldsymbol{x})=\int d k \check{f}(k) e^{i k_{\mu} \boldsymbol{x}^{\mu}},
$$

where

$$
\check{f}(k)=\frac{1}{(2 \pi)^{4}} \int d x f(x) e^{-i k_{\mu} x^{\mu}}
$$

is the usual Fourier transform. The idea is to replace the plane waves with their quantised counterparts. Note that $\bar{f}(\boldsymbol{x})=f(\boldsymbol{x})^{*}$, where $\bar{f}$ is the function obtained by pointwise complex conjugation, and ${ }^{*}$ means adjoint (as an operator). In particular $f(\boldsymbol{x})$ is selfadjoint if $f$ is real.

Note that, in order that both integrals are well defined, we require that both $f, \check{f}$ are $L^{1}$; such functions are called symbols; in particular the coordinate functions $x^{\mu}$ are not in the domain of the quantisation.

Since the map $f \mapsto f(\boldsymbol{x})$ is injective, it is possible to define an associative product of symbols by setting

$$
(f \star g)(\boldsymbol{x})=f(\boldsymbol{x}) g(\boldsymbol{x}),
$$

where on the right hand side the operator product is taken. Standard computations using (3.2) and antisymmetry of $\theta$ yield

$$
f(\boldsymbol{x}) g(\boldsymbol{x})=(f \star g)(\boldsymbol{x})=\widehat{f \tilde{\times}} g(\boldsymbol{x}),
$$

where

$$
(\check{f} \tilde{\times} \check{g})(k)=\int d h \check{f}(h) \check{g}(k-h) e^{-\frac{i}{2} h_{\mu} \theta^{\mu \nu} k_{\nu}} .
$$

More explicitly,

$$
(f \star g)(x)=\int d k e^{i k_{\mu} x^{\mu}} \int d h \check{f}(h) \check{g}(k-h) e^{-\frac{i}{2} h_{\mu} \theta^{\mu \nu} k_{\nu}} .
$$

The product $\star$ is called the twisted product; $\tilde{x}$ is the twisted convolution. 
The algebra $\mathcal{E}_{\theta}$ of symbols equipped with the product $\star$ and the involution $f \mapsto \bar{f}$ is a well defined, associative $*$-algebra. Note that only the relations (3.2) were used to define it; at this stage it is not necessary to know the precise form of the operators $\boldsymbol{x}^{\mu}$. However, knowing that they exist and are unique is sufficient to conclude that this $*$-algebra admits a unique $C^{*}$-completion, which is found to be the $C^{*}$-algebra $\mathcal{K}$ of compact operators, see [7].

Note that the $C^{*}$-completion of $\mathcal{E}_{\theta}$ is the same for all $\theta$ 's as above; hence the particular choice of $\theta$ is visible only in the quantisation prescription (3.3), while the $C^{*}$-algebra is the same for all choices.

Finally, observe that equation (1.1) is meaningless in this framework. For unbounded operators, even the operator product is a concept of little interest in general, because of problems with domains (operator product is composition of maps). Hence the notation $\boldsymbol{x}^{\mu} \star \boldsymbol{x}^{\nu}$ can not even be given a sound meaning as a product of operators. Only the Weyl relations (3.2) are unambiguously meaningful; in this context (3.1) is always understood as a symbolic reminder of the Weyl relations.

\subsection{Moyal expansion}

Let $f, g$ be symbols such that $\check{f}, \check{g}$ have compact support. Note that by the Paley-Wiener theorem this implies that $f, g$ are entire analytic functions of $\mathbb{R}^{4}$. It is important to keep in mind that they also are symbols, thus $L^{1}$; in particular, polynomials are ruled out.

We may now replace the exponential $\exp \left(-i \theta^{\mu \nu} h_{\mu} k_{\nu}\right)$ by its series expansion; for the selected subclass of symbols, $\int \sum=\sum \int$, and

$$
(\check{f} \times \check{g})=\sum_{n=0}^{\infty} \frac{(-i / 2)^{n}}{n !} \int d k\left(h_{\mu} \theta^{\mu \nu} k_{\nu}\right)^{n} \check{f}(k) \check{g}(k) .
$$

By standard Fourier theory

$$
(f \star g)(x)=\widehat{\check{f} \times \check{g}}(x)=\sum_{n=0}^{\infty} \frac{(i / 2)^{n}}{n !} m\left(\left(\theta^{\mu \nu} \partial_{\mu} \otimes \partial_{\nu}\right)^{n} f \otimes g\right)(x),
$$

where $m(f \otimes g)(x)=f(x) g(x)$. As a shorthand of the above (Moyal) expansion, one writes

$$
f \star g=m \circ e^{\frac{i}{2} \theta^{\mu \nu} \partial_{\mu} \otimes \partial_{\nu}} f \otimes g,
$$

which is unambiguously true for real analytic symbols.

The Moyal expansion is often said asymptotic. However this refers to the asymptotic behaviour of the truncated series when $\theta$ goes to zero; see [15]. There is an extensive literature about extensions of the Moyal expansion to wider classes of functions than real-analytic symbols. However, the underlying philosophy there - as far as I know - is to focus the attention on more general, standalone non local products, without correspondingly enlarging the domain of the quantisation. For this reason we will not discuss them in this review.

\subsection{Algebraic relations}

Usually the $*$-algebra generated by a finite set of elements with given commutation relations and adjoints is defined as the free algebra generated by that set, divided by the equivalence relation induced by the given commutation relations. However in our case there is a simpler way.

Consider the ring $\mathbb{C}\left[\boldsymbol{x}^{\mu}\right]$ of polynomials in the variables $\boldsymbol{x}^{0}, \boldsymbol{x}^{1}, \boldsymbol{x}^{2}, \boldsymbol{x}^{3}$. With $f g$ the usual product of two polynomials $f, g$, define the map

$$
m: f \otimes g \mapsto f g .
$$


The expression

$$
\mathcal{F}_{\theta}=e^{\frac{i}{2} \theta^{\mu \nu} \partial_{\mu} \otimes \partial_{\nu}}
$$

gives a well defined linear operator on $\mathbb{C}\left[x^{\mu}\right] \otimes \mathbb{C}\left[x^{\mu}\right]$, since for any two polynomials $f, g \in \mathbb{C}\left[x^{\mu}\right]$, only a finite number of terms in the sum

$$
\mathcal{F}_{\theta} f \otimes g=\sum_{n=0}^{\infty} \frac{(i / 2)^{n}}{n !}\left(\theta^{\mu \nu} \partial_{\mu} \otimes \partial_{\nu}\right)^{n} f \otimes g
$$

is different from 0 .

We now may set

$$
f \star g=m \circ F_{\theta} f \otimes g .
$$

One may directly check that the above defines an associative product of polynomials. Moreover, due to the antisymmetry of $\theta$, it is also involutive under the usual involution of polynomials (complex conjugation of coefficients). The unital $*$-algebra $\mathcal{A}_{\theta}$ obtained by equipping $\mathbb{C}\left[x^{\mu}\right]$ with the usual involution and the product $\star$ is generated by its elements $\boldsymbol{x}^{\mu}$, which are selfadjoint and fulfil

$$
\boldsymbol{x}^{\mu} \star \boldsymbol{x}^{\nu}-\boldsymbol{x}^{\nu} \star \boldsymbol{x}^{\mu}=i \theta^{\mu \nu}
$$

Since the algebra generated by a finite set of relations is unique (up to isomorphisms), here we are.

Notations: $\boldsymbol{x}^{\mu_{1}} \cdots \boldsymbol{x}^{\mu_{k}}$ is the product taken in $\mathbb{C}\left[\boldsymbol{x}^{\mu}\right]$; while $\boldsymbol{x}^{\mu_{1}} \star \cdots \star \boldsymbol{x}^{\mu_{k}}$ is taken in $\mathcal{A}_{\theta}$. Note in particular that, due to antisymmetry of $\theta,\left(\boldsymbol{x}^{\mu}\right)^{n}=\left(\boldsymbol{x}^{\mu}\right)^{\star n}$; moreover, $\boldsymbol{x}^{\mu} \star \boldsymbol{x}^{\nu}=\boldsymbol{x}^{\mu} \boldsymbol{x}^{\nu}+(i / 2) \theta^{\mu \nu}$.

This algebra contains in particular the elements $\boldsymbol{x}^{\nu}$, and thus admits no $C^{*}$-completion, as discussed in the first part of this section.

It is easily seen that the (maximal) degree of polynomials is additive under the deformed product $\star$ (the non-commutative corrections $f \star g-f g$ being of lower degree). Hence, like in $\mathbb{C}\left[\boldsymbol{x}^{\mu}\right]$, no polynomial of non zero degree can be invertible in $\mathcal{A}_{\theta}$. It follows that any element on nonzero degree has the whole complex plane as its spectrum (because resolvents never exist), no matter whether it is selfadjoint, skewadjoint, or none of the two. Moreover, since the only invertible elements are the multiples of the identity, the only unitary elements in $\mathcal{A}_{\theta}$ are the complex phases.

Note that in particular

$$
\operatorname{spec}_{\mathcal{A}_{\theta}}\left(\boldsymbol{x}^{\mu}\right)=\mathbb{C},
$$

which should raise some serious concerns about the physical interpretation of such coordinates under a quantum perspective.

\subsection{Dangers of the Moyal expansion}

We see that we are facing a confusing situation. The same expression

$$
m\left(e^{(i / 2) \theta^{\mu \nu} \partial_{\mu} \otimes \partial_{\nu}} f \otimes g\right)
$$

can be used for pairs $f, g$ of polynomials in $\mathcal{A}_{\theta}$ or of functions in a dense sub algebra of $\mathcal{E}_{\theta}$ (not containing the polynomials); in both cases it gives the right product in the corresponding algebra, which in both cases is denoted by $\star$. However the two algebras are dramatically non isomorphic. 
One common misuse is to understand $\star$ as a product in $\mathcal{A}_{\theta}$ (for example, writing down (1.1)), yet making use of the Weyl quantisation prescription, which only makes sense in $\mathcal{E}_{\theta}$. The two are mutually excluding, as we have seen. Formal computations under such a mixed formalism are somewhat out of control, since one may inadvertently switch from an algebra to the other, and back; the result might depend on the way the computation is done.

Let us see another example of what happens if one does not specify where the symbols live:

"Let $f, g$ be functions with disjoint supports, and consider their Moyal $\star$-product

$$
f \star g=m\left(e^{(i / 2) \theta^{\mu \nu} \partial_{\mu} \otimes \partial_{\nu}} f \otimes g\right) .
$$

Since derivatives cannot enlarge the domains, at any order the product vanishes, and $f \star g=0 . "$

In the best case, this statement is a trivial tautology (whatever of the two algebras we choose to work with): if two real-analytic symbols or two polynomials have disjoint supports, at least one of the two is zero, so that their product is evidently zero; which is certainly true, yet not so thrilling.

If $f, g$ are generic $\mathcal{C}^{\infty}$ functions, the above statement is meaningless, since in general the Moyal expansion does not converge and there is no $f \star g$ at all. One solution is to understand the Moyal product in the sense of formal power series in $\theta$; in this direction, see e.g. [16], and references therein (especially to work of Drinfel'd and Kontsevich). However, doing this one misses something important from the point of view of nonlocality. Indeed, the integral form of the twisted product in $\mathcal{E}_{\theta}$ is intrinsically non local. On the contrary, the structure of the Moyal expansion alone is unable to reproduce the non local character of the full product: for generic functions, it is non-commutative but local (look at the supports) at any order ${ }^{11}$. Only when applied to real analytic symbols is the Moyal expansion able to catch the full non local content of the twisted product; for, in the analytic world (as in the stock exchange), local is global.

Finally, quite common a misuse is to formally use the Moyal expansion as a "product" of functions on unspecified type, in the spirit of "existence in notation". As it is easy to imagine, this also is completely out of control. A spectacular example is the famous saga of (non existing) "violations of unitarity", which will be discussed in some detail in Section 6, and especially in Section 6.4. We also will see that Filk diagrams and rules can be obtained in this way.

Let me conclude with a point of history. Weyl quantisation was suggested, in the framework of canonical quantisation, by Hermann Weyl in his famous book [17]; everybody interested in this field should find the time for reading those beautiful pages. Weyl also advocated the usefulness of symbolic calculus; however, he did not write down the explicit formulas. The first appearance of the deformed product is in the famous paper of von Neumann, on the uniqueness of Schrödinger operators [18]. However, for technical reasons, he decided to work in Fourier space, so that he only wrote down the twisted convolution product. Wigner found the solution of the inverse problem (given the operator, find the corresponding symbol), while he was working to his (unsuccessful) theory of negative probabilities applied to quantum physics. His follower Moyal wrote down (the quantum mechanical version of) the tricky expansion we discussed in Section 3.2. The correct integral formula for the twisted product in configuration space first appeared in printed form in papers by Baker and Pool (see references of [15]). Finally, the covariant version of the Weyl quantisation (in terms of the quantum coordinates of the spacetime, instead of the Schrödinger coordinates of the phase space) and the corresponding Weyl calculus was first suggested by Doplicher, Fredenhagen and Roberts [7].

\footnotetext{
${ }^{11}$ By the way, this also provides a proof that geometric quantisation is not equivalent to Weyl quantisation, since the resulting algebras are deeply inequivalent.
} 


\subsection{Existence of representations}

We shortly recall the very simple, yet effective argument of [7] for the existence of the unbounded operators associated to the quantisation; for more general choices of $\theta$ and for time/space commutative models, see Section 5.1). Let $\Lambda$ be a $4 \times 4$ matrix (not necessarily in the Lorentz group); let moreover ${ }^{12}$

$$
S=\left(S^{\mu \nu}\right)=\left(\begin{array}{cccc}
0 & 0 & -1 & 0 \\
0 & 0 & 0 & -1 \\
1 & 0 & 0 & 0 \\
0 & 1 & 0 & 0
\end{array}\right) .
$$

Then define

$$
\begin{aligned}
& \boldsymbol{X}^{0}=\boldsymbol{P}_{1}, \\
& \boldsymbol{X}^{1}=\boldsymbol{P}_{2}, \\
& \boldsymbol{X}^{2}=\boldsymbol{Q}_{1}, \\
& \boldsymbol{X}^{3}=\boldsymbol{Q}_{2},
\end{aligned}
$$

where $\boldsymbol{P}_{j}=-i \partial_{j}$ and $\boldsymbol{Q}_{j}=s_{j}$ are the usual Schrödinger operators on $\mathfrak{H}=L^{2}\left(\mathbb{R}^{2}, d s_{1} d s_{2}\right)$ for the particle on the plane, fulfilling $\left[\boldsymbol{P}_{j}, \boldsymbol{Q}_{k}\right]=-i \delta_{j k} \boldsymbol{I}$ strongly (this is a purely formal analogy, with no physical interpretation). Then clearly the Weyl form of the relations

$$
\left[\boldsymbol{X}^{\mu}, \boldsymbol{X}^{\nu}\right]=i S^{\mu \nu} \boldsymbol{I}
$$

is fulfilled (as regular relations; see last paragraph of Section 3.1 at page 9). It follows that, for any matrix $\Lambda=\left(\Lambda_{\nu}^{\mu}\right)$ (not necessarily in the Lorentz group; but we use a covariant notation for later convenience), the operators

$$
\boldsymbol{x}^{\mu}=\Lambda_{\nu}^{\mu} \boldsymbol{X}^{\nu}
$$

fulfil

$$
\left[\boldsymbol{x}^{\mu}, \boldsymbol{x}^{\nu}\right]=i \theta^{\mu \nu} \boldsymbol{I}
$$

in the Weyl form (3.2), where

$$
\theta^{\mu \nu}=\Lambda_{\mu^{\prime}}^{\mu} \Lambda_{\nu^{\prime}}^{\nu} S^{\mu^{\prime} \nu^{\prime}} .
$$

This gives at once the class of matrices $\theta$ for which the argument works, and existence of the corresponding operators $\boldsymbol{x}^{\mu}$.

Note also that if in addition $\Lambda$ is invertible then, given the operators $\boldsymbol{x}^{\mu}$, we may reconstruct the Schrödinger operators $\boldsymbol{P}_{j}, \boldsymbol{Q}_{j}$. Hence in this case we also know that there cannot be other irreducible representations (up to equivalence), as a corollary of von Neumann uniqueness of Schrödinger operators [18].

\section{Translation covariant representations}

For the special case of $\theta=S$, let us see how to construct a translation covariant representation. The idea is to consider other two independent Schrödinger pairs $\boldsymbol{P}_{3}, \boldsymbol{Q}_{3}, \boldsymbol{P}_{4}, \boldsymbol{Q}_{4}$, so that $\left[\boldsymbol{P}_{j}, \boldsymbol{Q}_{k}\right]=i \delta_{j k} \boldsymbol{I}, j, k=1,2,3,4$. Now take $\boldsymbol{X}^{\mu}$ as in (3.8); moreover, take

$$
\Pi_{0}=Q_{1}+Q_{3},
$$

\footnotetext{
${ }^{12}$ The matrix $S$ was denote $\sigma_{0}$ in [7]. The change of notation is motivated by the fact that here we use repeated sum over dummy indices instead of matrix notation.
} 


$$
\begin{aligned}
& \boldsymbol{\Pi}_{1}=\boldsymbol{Q}_{2}+\boldsymbol{Q}_{4}, \\
& \boldsymbol{\Pi}_{2}=\boldsymbol{P}_{3}-\boldsymbol{P}_{1}, \\
& \boldsymbol{\Pi}_{3}=\boldsymbol{P}_{4}-\boldsymbol{P}_{2},
\end{aligned}
$$

and $\boldsymbol{\Pi}^{\mu}=g^{\mu \nu} \boldsymbol{\Pi}_{\nu}$. It is easy to check that

$$
\left[\boldsymbol{\Pi}^{\mu}, \boldsymbol{\Pi}^{\nu}\right]=0, \quad\left[\boldsymbol{\Pi}^{\mu}, \boldsymbol{X}^{\nu}\right]=-i g^{\mu \nu} \boldsymbol{I}
$$

hence the $\Pi^{\mu}$ 's are the pairwise commuting generators of spacetime translations (in this particular representation). Note that the localisation algebra has no dynamical content by itself, so that these generators can not be interpreted as the "momenta" of some theory. A similar trick works for every invertible $\theta$, see $[7]^{13}$.

\section{Covariance and the DFR model}

We now will describe the DFR model [7]. We will take a bottom-up strategy. Firstly, we will try to cure the lack of covariance by a simple construction, and obtain a class of $C^{*}$-algebras which are covariant under the action of the full Poincarè group. Then we will select a particular algebra in this class (corresponding to a particular orbit of the antisymmetric tensors under the action of the Lorentz group). Finally we will show that this algebra fulfils physically motivated uncertainty relations.

This line of exposition does not make justice to the original paper, where the structure was derived from very general assumptions and ansätze (top-down approach), instead of being constructed by hand. However I hope that this will make easier, for a broad readership, to grasp in a few pages some of the more delicate technical aspects. Moreover, the comparison with the usual approach to the so called "canonical quantum spacetime" will be more transparent. The price to pay is that some apparently arbitrary assumptions will have to find their motivations only in the end.

In what follows, we will assume that $\theta$ is such that there exists a unique representation of the Weyl relations (3.2). Moreover, we will keep separate the purely algebraic structure and the choice of the length scale (or unit of measure) by setting

$$
\theta^{\mu \nu}=\lambda^{2} \sigma^{\mu \nu},
$$

where $\lambda$ is a length and the entries of $\sigma$ are pure numbers.

Correspondingly, we will write $\star_{\sigma}$ instead of $\star$ for the product in $\mathcal{E}_{\sigma}$; the symbol $\star$ will be reserved for a more general product. Hence, we may rewrite (3.7) as

$$
\left(f \star_{\sigma} g\right)(x)=\int d k e^{i k_{\mu} x^{\mu}} \int d h \check{f}(h) \check{g}(k-h) e^{-i \frac{\lambda^{2}}{2} h_{\mu} \sigma^{\mu \nu} k_{\nu}} .
$$

\section{1 "Be wise, covariantise"}

For a given $L=(\Lambda, a)$ in the Poincaré group $\mathscr{P}$, let

$$
\begin{aligned}
& f^{\prime}(x)=f\left(\Lambda^{-1}(x-a)\right), \quad g^{\prime}(x)=g\left(\Lambda^{-1}(x-a)\right), \\
& \left(f \star_{\sigma} g\right)^{\prime}(x)=\left(f \star_{\sigma} g\right)\left(\Lambda^{-1}(x-a)\right) .
\end{aligned}
$$

Of course, the product $\star_{\sigma}$ is not covariant: in general, we have

$$
f^{\prime} \star_{\sigma} g^{\prime} \neq\left(f \star_{\sigma} g\right)^{\prime} \text {. }
$$

\footnotetext{
${ }^{13}$ There, the conjugate Hilbert space is used to get compact formulas; note that if $(\boldsymbol{J} \psi)(s)=\overline{\psi(s)}$ is the usual conjugation on $L^{2}(\mathbb{R}, d s)$, then $\boldsymbol{J} \boldsymbol{P} \boldsymbol{J}=-\boldsymbol{P}$.
} 
However (as one can easily imagine) a simple computation yields

$$
\left(f^{\prime} \star_{\sigma^{\prime}} g^{\prime}\right)(x)=\left(f \star_{\sigma} g\right)^{\prime}(x),
$$

where

$$
\sigma^{\prime \mu \nu}=\Lambda_{\mu^{\prime}}^{\mu} \Lambda_{\nu^{\prime}}^{\nu} \sigma^{\mu^{\prime} \nu^{\prime}}
$$

This suggests a very simple way out. The idea is to turn the parameter $\sigma$ into a variable. Let $\Sigma$ be a set of antisymmetric matrices which is stable under the action

$$
\Lambda: \sigma \mapsto \sigma^{\prime}=\left(\Lambda_{\mu^{\prime}}^{\mu} \Lambda_{\nu^{\prime}}^{\nu} \sigma^{\mu^{\prime} \nu^{\prime}}\right)
$$

Then consider generalised symbols, namely functions of $\Sigma \times \mathbb{R}^{4}$, and define the product

$$
(f \star g)(\sigma ; x)=\int d k e^{i k_{\mu} x^{\mu}} \int d h \check{f}(\sigma ; h) \check{g}(\sigma ; k-h) e^{-i \frac{\lambda^{2}}{2} h_{\mu} \sigma^{\mu \nu} k_{\nu}},
$$

where of course $\check{f}$ is the Fourier transform of $f(\sigma ; \cdot)$ for each $\sigma$ fixed. In addition, set pointwise complex conjugation as the involution:

$$
f^{\star}(\sigma ; x)=\overline{f(\sigma ; x)} .
$$

The above gives a well defined $*$-algebra, which we denote $\mathcal{E}^{(0)}$.

If we define the action of $L=(\Lambda, a) \in \mathscr{P}$, the Poincaré group, by

$$
\begin{aligned}
& (\gamma(L) f)\left(\sigma^{\prime} ; x^{\prime}\right)=\operatorname{det} \Lambda f(\sigma ; x), \\
& \sigma^{\prime \mu \nu}=\Lambda_{\mu^{\prime}}^{\mu} \Lambda_{\nu^{\prime}}^{\nu} \sigma^{\mu^{\prime} \nu^{\prime}}, \quad x^{\prime}=\Lambda x+a,
\end{aligned}
$$

then by construction we obtain covariance:

$$
\gamma(L)(f \star g)=(\gamma(L) f) \star(\gamma(L) g) .
$$

In more technical language, for each $L, \gamma(L)$ is a $*$-automorphism of $\mathcal{E}^{(0)}$, and $\gamma\left(L_{1}\right) \gamma\left(L_{2}\right)=$ $\gamma\left(L_{1} L_{2}\right)$; this situation is often described in the mathematical literature as $\gamma$ providing an action of $P$ by automorphisms of $\mathcal{E}^{(0)}$.

We still have to fix the class of functions, and the set $\Sigma$. Of course, for every $\sigma$ fixed we have to require that $f(\sigma ; \cdot)$ is an admissible symbol. As for $\Sigma$, the simplest choice is to pick a single orbit in the space of antisymmetric matrices under the given action. For the moment this choice only can be motivated by the quest for simplicity; but we will see that it corresponds to assume that there is one only characteristic length driving the algebraic structure.

For the purpose of computations, the above is almost all one needs. However, since we put emphasis on the importance of existence of $C^{*}$-completions (especially in order to have a non trivial theory from the spectral point of view), let us give a hint about the underlying $C^{*}$ structure, and how to unveil it.

Giving a closer look at the way the product of generalised symbols is defined, we may see that there is a natural bundle structure emerging: indeed, the product

$$
(f \star g)(\sigma ; \cdot)=f(\sigma ; \cdot) \star_{\sigma} g(\sigma ; \cdot), \quad \sigma \in \Sigma,
$$

appears as a fibrewise product over the base space $\Sigma$. This suggest to take $f$ as a continuous function (vanishing at infinity) sending each $\sigma \in \Sigma$ to an element of $\mathcal{E}_{\sigma}$, namely a continuous section of a bundle of algebras. At a first glance, it seems to be a non trivial bundle, since each fibre is different; however we now can remember that every fibre is dense in the same 
$C^{*}$-completion, which is the algebra $\mathcal{K}$ of compact operators on the separable Hilbert space. Hence we are naturally led to consider a trivial bundle of $C^{*}$-algebras, with base space $\Sigma$ and standard fibre $\mathcal{K}$. It may be shown (see [7]) that actually

$$
\mathcal{E}=\mathcal{C}_{0}(\Sigma, \mathcal{K})
$$

is the unique $C^{*}$-completion of the algebra $\mathcal{E}^{(0)}$ of continuous sections (= generalised symbols).

Note that the action $\gamma$ of the Poincaré group on the algebra of continuous sections maps each fibre over $\sigma$ onto the fibre over $\sigma^{\prime}$ and extends by continuity to an action by automorphisms of the full $C^{*}$-algebra $\mathcal{E}$.

\subsection{Representations}

In this section, we describe how to construct the representations of the $C^{*}$-algebra $\mathcal{E}=\mathcal{C}_{0}(\Sigma, \mathcal{K})$, using the Dirac notation; for a mathematically rigorous treatment, see the original paper.

Let us fix $\Sigma$ to be the orbit containing the matrix $S$ described at the end of Section 3. This is not technically necessary at this point of the discussion, but it simplifies the notations, and we will anyhow find that this choice is the correct one. We also fix the choice $\lambda=\lambda_{P}$, the Planck length.

The reader should have clear in mind the idea underlying the construction described in Section 3.5.

We consider the Hilbert space $^{14}$

$$
\mathfrak{H}=L^{2}(\mathscr{L}, d \Lambda) \otimes L^{2}\left(\mathbb{R}^{2}, d s_{1} d s_{2}\right),
$$

where $d \Lambda$ is the Haar measure of the Lorentz group $\Lambda$. As usual, we associate to it a complete set of generalised kets

$$
|\Lambda\rangle\left|s_{1}, s_{2}\right\rangle, \quad \Lambda \in \mathscr{L},\left(s_{1}, s_{2}\right) \in \mathbb{R}^{2},
$$

with bracket

$$
\left\{\left\langle\Lambda\left|\left\langle s_{1}, s_{2}\right|\right\}\left\{\left|\Lambda^{\prime}\right\rangle\left|s_{1}^{\prime}, s_{2}^{\prime}\right\rangle\right\}=\left\langle\Lambda \mid \Lambda^{\prime}\right\rangle\left\langle s_{1}, s_{2} \mid s_{1}^{\prime}, s_{2}^{\prime}\right\rangle=\delta\left(\Lambda^{-1} \Lambda^{\prime}\right) \delta\left(s_{1}-s_{1}^{\prime}\right) \delta\left(s_{2}-s_{2}^{\prime}\right),\right.\right.
$$

where integrals are taken with the measure $d \Lambda d s_{1} d s_{2}$ and $\delta(\Lambda) d \Lambda$ is the purely atomic normalised measure on $\mathscr{L}$, concentrated on $I$.

We define the operators $\boldsymbol{q}^{\mu}$ by their actions on the kets $|\Lambda\rangle|\xi\rangle$ :

$$
\boldsymbol{q}^{\mu}|\Lambda\rangle|\xi\rangle=\lambda_{P}|\Lambda\rangle\left\{\Lambda^{\mu}{ }_{\nu} \boldsymbol{X}^{\nu}|\xi\rangle\right\},
$$

where the $\boldsymbol{X}^{\mu}$ are described in Section 3.5. These operators being defined in terms of the Schrödinger operators, we have

$$
\operatorname{spec}\left(\boldsymbol{q}^{\mu}\right)=\mathbb{R} .
$$

We may easily check that the operators $Q^{\mu \nu}$ defined by

$$
\left[\boldsymbol{q}^{\mu}, \boldsymbol{q}^{\nu}\right]=i \lambda_{P}^{2} \boldsymbol{Q}^{\mu \nu}
$$

are simultaneously diagonalised by the kets $|\Lambda\rangle\left|s_{1}, s_{2}\right\rangle$ :

$$
Q^{\mu \nu}|\Lambda\rangle|\xi\rangle=\Lambda_{\mu^{\prime}}^{\mu} \Lambda_{\nu^{\prime}} S^{\mu^{\prime} \nu^{\prime}}|\Lambda\rangle|\xi\rangle .
$$

\footnotetext{
${ }^{14}$ If there were a Lorentz invariant measure $d \sigma$ on $\Sigma$, we could have taken kets of the form $|\sigma\rangle|\xi\rangle$ and integrate with $d \sigma$ instead of $d \Lambda$. It is a fact of life that such a measure does not exist.
} 
It follows that the spectrum of each $Q^{\mu \nu}$ is

$$
\operatorname{spec}\left(\boldsymbol{Q}^{\mu \nu}\right)=\left\{\sigma^{\mu \nu}: \sigma \in \Sigma\right\}=\mathbb{R} .
$$

We have a unitary representation $\boldsymbol{U}$ of the Lorentz group

$$
\boldsymbol{U}(\Lambda)|M\rangle\left|s_{1}, s_{2}\right\rangle=|\Lambda M\rangle\left|s_{1}, s_{2}\right\rangle ;
$$

it fulfils

$$
\begin{aligned}
& \boldsymbol{U}(\Lambda)^{-1} \boldsymbol{q}^{\mu} \boldsymbol{U}(\Lambda)=\Lambda^{\mu}{ }_{\nu} \boldsymbol{q}^{\nu}, \\
& \boldsymbol{U}(\Lambda)^{-1} \boldsymbol{Q}^{\mu \nu} \boldsymbol{U}(\Lambda)=\Lambda^{\mu}{ }_{\mu^{\prime}} \Lambda_{\mu^{\prime}} \boldsymbol{Q}^{\mu^{\prime} \nu^{\prime}} .
\end{aligned}
$$

Let now $f=f(\sigma ; x)$ be a generalised symbol, as described in Section 4.1. We may define its DFR quantisation à la Weyl

$$
f(\boldsymbol{Q} ; \boldsymbol{q})|\Lambda\rangle|\xi\rangle=|\Lambda\rangle\left\{\int d k \check{f}\left(\left(\Lambda^{\mu}{ }_{\mu^{\prime}} \Lambda^{\nu}{ }_{\nu^{\prime}} S^{\mu^{\prime} \nu^{\prime}}\right) ; k\right) e^{i \lambda_{P} k_{\mu} \Lambda^{\mu}{ }_{\nu} \boldsymbol{X}^{\nu}}|\xi\rangle\right\} .
$$

Note that if we take a function of the form

$$
f(\sigma ; x)=\left(f_{1} \otimes f_{2}\right)(\sigma ; x)=f_{1}(\sigma) f_{2}(x),
$$

then the DFR quantisation prescription gives the operator product

$$
\left(f_{1} \otimes f_{2}\right)(\boldsymbol{Q} ; \boldsymbol{q})=f_{1}(\boldsymbol{Q}) \int d k \check{f}_{2}(k) e^{i k \boldsymbol{q}},
$$

where $f_{1}(\boldsymbol{Q})$ is the usual evaluation of a function on a set of pairwise commuting operators (joint functional calculus). By a direct check, we find in general

$$
f(\boldsymbol{Q} ; \boldsymbol{q}) g(\boldsymbol{Q} ; \boldsymbol{q})=(f \star g)(\boldsymbol{Q} ; \boldsymbol{q}), \quad \bar{f}(\boldsymbol{Q} ; \boldsymbol{q})=f(\boldsymbol{Q} ; \boldsymbol{q})^{*}
$$

and

$$
\boldsymbol{U}(L) f(\boldsymbol{Q} ; \boldsymbol{q}) \boldsymbol{U}(L)^{-1}=(\gamma(L) f)(\boldsymbol{Q} ; \boldsymbol{q}), \quad L \in \mathscr{P} .
$$

Finally, we may consider the functions $\sigma^{\mu \nu} \sigma_{\mu \nu}$ and $\left(\sigma^{\mu \nu}(* \sigma)_{\mu \nu}\right)^{2}$ of $\Sigma$, where $(* \sigma)_{\mu \nu}=$ $\frac{1}{2} \epsilon_{\mu \nu \rho \tau} \sigma^{\rho \tau}$ is the Hodge dual of $\sigma$. These functions are invariants of the full Lorentz group, and there are no other invariants independent from these two. Since they are invariants, their values are constant and can be computed at any point in $\Sigma$, e.g. at $S$. Hence

$$
\sigma^{\mu \nu} \sigma_{\mu \nu}=0, \quad\left(\sigma^{\mu \nu}(* \sigma)_{\mu \nu}\right)^{2}=16, \quad \sigma \in \Sigma .
$$

It follows that the same relations hold true with $Q$ in the place of $\sigma$.

The Lorentz covariant representation $\left(\boldsymbol{q}^{\mu}, \boldsymbol{U}\right)$ described above is essentially (i.e. up to equivalence and multiplicity) unique. This follows from a "fibrewise" von Neumann uniqueness argument $^{15}[7]$.

An adaptation of the same argument, where now we take the translation covariant representations described at the end of Section 3.5 as building blocks, will give a full Poincaré covariant representation.

\footnotetext{
${ }^{15}$ The reader may recognise in the above construction a direct integral over $\mathscr{L}$ of irreducible representations labeled by $\sigma=\Lambda S \Lambda^{t}$. For generic kets $|\psi\rangle \in \mathfrak{H}$ of finite length, the functions $\psi\left(\Lambda ; s_{1}, s_{2}\right)=\left\{\left\langle\Lambda\left|\left\langle s_{1}, s_{2}\right|\right\} \mid \psi\right\rangle\right.$ may be recognised as the measurable fields $\Lambda \mapsto \psi(\Lambda ; \cdot)$ in the direct integral $\mathfrak{H}=\int{ }^{\oplus} d \Lambda L^{2}\left(\mathbb{R}^{2}\right)$.
} 


\subsection{The DFR model recovered}

In the preceding section we have shown that the universal, covariant representation of the $C^{*}$ algebra $\mathcal{C}_{0}(\Sigma, \mathcal{L})$ is associated with selfadjoint operators $\boldsymbol{q}^{\mu}$ fulfilling

$$
\left[\boldsymbol{q}^{\mu}, \boldsymbol{q}^{\nu}\right]=i \lambda_{P}^{2} \boldsymbol{Q}^{\mu \nu}
$$

where

$$
\begin{aligned}
& {\left[\boldsymbol{q}^{\mu}, \boldsymbol{Q}^{\nu \rho}\right]=0,} \\
& \boldsymbol{Q}^{\mu \nu} \boldsymbol{Q}_{\mu \nu}=0, \\
& \left(\boldsymbol{Q}^{\mu \nu}(* \boldsymbol{Q})_{\mu \nu}\right)^{2}=16 \boldsymbol{I} .
\end{aligned}
$$

In [7], the above relations were the starting point for the analysis. Indeed, the pattern proposed there was precisely opposite to the one we followed.

So, everything is a consequence of the relations (4.2) and their full Poincaré covariance expressed by

$$
\begin{aligned}
& \boldsymbol{U}(\Lambda, a)^{-1} \boldsymbol{q}^{\mu} \boldsymbol{U}(\Lambda, a)=\Lambda_{\nu}^{\mu}{ }_{\nu} \boldsymbol{q}^{\nu}+a^{\mu} \boldsymbol{I} \\
& \boldsymbol{U}(\Lambda, a)^{-1} \boldsymbol{Q}^{\mu \nu} \boldsymbol{U}(\Lambda, a)=\Lambda_{\mu^{\prime}}^{\mu} \Lambda_{\mu^{\prime}}^{\mu} \boldsymbol{Q}^{\mu^{\prime} \nu^{\prime}}
\end{aligned}
$$

for $(\Lambda, a) \in \mathscr{P}$, the Poincaré group.

But where do they come from? To give a quantitative content to the remarks of Section 2, in [7] some important steps were made precise.

- The (limited) scope of the model: an idealised situation describing scattering experiments in particle physics. This means that the density of processes is so low that it does not produce significant deviations from the flat geometry of the laboratory; however their energy is so high that deviations from classical flat spacetime are produced only at very small scales.

- The necessity of consistence with usual particle physics (described in terms of quantum fields). This requires in particular that the usual classical (commutative) Minkowski spacetime must be obtained in the large scale limit (possibly at the cost of extra dimensions).

- The necessity of ordinary Poincaré covariance (phrased in the dear old Wigner's language for quantum symmetries). This for many reasons: a) the need for preserving Wigner's classification of particles scattered far away (which are then detected in the "classical region", where non-commutativity is smeared away: in- and out-fields are defined on the classical spacetime); b) the remark that transformations of reference frames are global and affect the cosmic scale as well as the Planck scale; c) the need for preserving the symmetry between active and passive point of view (think of an experiment in a laboratory).

Within this conceptual framework, we may formulate the first DFR problem:

(DFR1) Find relations between the uncertainties $\Delta x^{\mu}$, which prevent the formation of trapped surfaces enclosing the observed region, as an effect of localisation alone.

Note that, in view of the limited scope of the model, dynamical black hole formation is prevented from the outset, since the large scale geometric background is fixed, and it is flat. In a wouldbe, still unknown more general model, one should be able to distinguish dynamical black hole formation from localisation-induced black hole formation, and find conditions preventing the latter only. 
It is possible to carry over a heuristic analysis, where localisation states are modeled by coherent states of the form $e^{i \phi(f)}|0\rangle(|0\rangle$ is the Fock vacuum for a free field $\phi)$. If the ("commutative") test function $f$ has support in a 4-box of sides $\Delta x^{\mu}$, bounds from Einstein equations are obtained by (crude) estimates on the expectation of the energy-momentum tensor in such states. We omit the details, for which the reader is referred to the original paper [7]. This leads to the relations

$$
\begin{aligned}
& \Delta x^{0}\left(\Delta x^{1}+\Delta x^{2}+\Delta x^{3}\right) \geqslant \lambda_{P}^{2}, \\
& \Delta x^{1} \Delta x^{2}+\Delta x^{1} \Delta x^{3}+\Delta x^{2} \Delta x^{3} \geqslant \lambda_{P}^{2} .
\end{aligned}
$$

The second DFR problem comes from the idea of following the pattern which led to canonical quantisation in the 1920s: given the uncertainty relations, find the commutation relations which reproduce them by the uncertainty theorem ${ }^{16}$.

(DFR2) Find commutation relations between selfadjoint operators $\boldsymbol{q}^{\mu}$ such that the relations (4.4) are fulfilled if one replaces $\Delta_{\omega} \boldsymbol{q}^{\mu}$ for $\Delta x^{\mu}$, no matter which state $\omega$ is chosen.

Above, $\Delta_{\omega} \boldsymbol{q}^{\mu}$ is the usual uncertainty of the operator $\boldsymbol{q}^{\mu}$ in the state $\omega$, namely

$$
\Delta_{\omega} \boldsymbol{q}^{\mu}=\sqrt{\omega\left(\left(\boldsymbol{q}^{\mu}\right)^{2}\right)-\omega\left(\boldsymbol{q}^{\mu}\right)^{2}} .
$$

Now it is clear that there is nothing bad in the uncertainty relations not having a covariant look'n feel since, despite the misleading notation, in general the uncertainties $\Delta_{\omega} \boldsymbol{q}^{\mu}$ are not the components of a covariant vector. Indeed $\Delta_{\omega}$ is not a linear functional on operators, so that $\Lambda_{\nu}^{\mu} \Delta_{\omega}\left(\boldsymbol{q}^{\nu}\right) \neq \Delta_{\omega}\left(\Lambda_{\nu}^{\mu} \boldsymbol{q}^{\nu}\right)$, in general. What is important is to check that the relations hold in any reference frame.

To solve problem DFR2, we observe that a measure of non-commutativity of $n$ elements is given by the determinant

$$
\left[\boldsymbol{A}_{1}, \boldsymbol{A}_{2}, \ldots, \boldsymbol{A}_{n}\right]:=\sum \epsilon_{j_{1} \ldots j_{n}} \boldsymbol{A}_{j_{1}} \cdots \boldsymbol{A}_{j_{n}}=\operatorname{det}\left[\begin{array}{cccc}
\boldsymbol{A}_{1} & \boldsymbol{A}_{2} & \ldots & \boldsymbol{A}_{n} \\
\boldsymbol{A}_{1} & \boldsymbol{A}_{2} & \ldots & \boldsymbol{A}_{n} \\
\vdots & \vdots & \ddots & \vdots \\
\boldsymbol{A}_{1} & \boldsymbol{A}_{2} & \ldots & \boldsymbol{A}_{n}
\end{array}\right]
$$

which for $n=2$ reduces to the usual commutator. Hence a model with 4 coordinates is fixed by giving conditions on

$$
\left[\boldsymbol{q}^{\mu}, \boldsymbol{q}^{\nu}\right], \quad\left[\boldsymbol{q}^{\mu}, \boldsymbol{q}^{\nu}, \boldsymbol{q}^{\rho}\right], \quad\left[\boldsymbol{q}^{\mu}, \boldsymbol{q}^{\nu}, \boldsymbol{q}^{\rho}, \boldsymbol{q}^{\tau}\right] .
$$

Setting

$$
(* \boldsymbol{Q})_{\mu \nu}=\frac{1}{2} \epsilon_{\mu \nu \rho \tau} \boldsymbol{Q}^{\rho \tau}
$$

we have

$$
\left[\boldsymbol{q}^{0}, \ldots, \boldsymbol{q}^{3}\right]=-\frac{1}{2} \boldsymbol{Q}^{\mu \nu}(* \boldsymbol{Q})_{\mu \nu}
$$

which is invariant under the special Lorentz group $\mathscr{L}_{+}^{\uparrow}$. There also is the full Lorentz invariant

$$
\boldsymbol{Q}^{\mu \nu} \boldsymbol{Q}_{\mu \nu}
$$

\footnotetext{
${ }^{16}$ We recall that, given two selfadjoint operators $\boldsymbol{A}, \boldsymbol{B}$, then for any state $\omega$ (which may be a vector state or a density matrix, it is irrelevant), we have $\Delta_{\omega}(\boldsymbol{A}) \Delta_{\omega}(\boldsymbol{B}) \geqslant \frac{1}{2}|\omega([\boldsymbol{A}, \boldsymbol{B}])|$.
} 
and there are no other invariants which are independent from these ones. Hence the only Poincaré invariant constrains which can be formulated with the determinants (4.5) must be given in terms of these objects and of $\left[\boldsymbol{q}^{\mu}, \boldsymbol{q}^{\nu}, \boldsymbol{q}^{\rho}\right]$.

With the simplifying ansatz $\left[\boldsymbol{q}^{\mu}, \boldsymbol{Q}^{\nu \rho}\right]=0$ (no physical motivations for it), which may be equivalently written as

$$
\left[\boldsymbol{q}^{\mu}, \boldsymbol{q}^{\nu}, \boldsymbol{q}^{\rho}\right]=0
$$

it is possible to show that the solution of problem DFR2 is provided precisely by (4.2).

Writing down commutation relations is not enough! Existence of operators actually fulfilling them is not granted; hence we have the third DFR problem

\section{(DFR3) Find operators $\boldsymbol{q}^{\mu}$ which fulf il the relations (4.2), and are covariant.}

This problem is solved precisely by the construction described in Section 4.2.

We close by observing that, in the DFR model, the spectrum of $\left[\boldsymbol{q}^{0}, \boldsymbol{q}^{1}, \boldsymbol{q}^{2}, \boldsymbol{q}^{3}\right]$ is $\left\{ \pm 2 \lambda_{P}^{4}\right\}$, while the spectrum of $\left[\boldsymbol{q}^{\mu}, \boldsymbol{q}^{\nu}\right]\left[\boldsymbol{q}_{\mu}, \boldsymbol{q}_{\nu}\right]$ is zero. Hence in this model there is one only universal length, which is precisely $\lambda_{P}$.

\subsection{Working with the DFR model}

Due to the uniqueness of the representation, it is useful to forget the difference between abstract elements and their representations. So, we think of $f(\boldsymbol{Q} ; \boldsymbol{q})$ both as of an operator, and of an element of the $C^{*}$-algebra; and, since the algebra of continuous sections is naturally embedded in its unique $C^{*}$-completion, we are allowed to think of $f(\boldsymbol{Q} ; \boldsymbol{q})$ as a function of $(\sigma, x)$. This way of reasoning may seem at first rather confusing, since one thinks of the same symbol as of an operator, a function, or an element of an abstract $C^{*}$-algebra depending on the convenience of the moment. However, it is quite a fruitful point of view.

To make this clear, let us work out some example which will be used in later sections.

Firstly, observe that, if $f=f(x)$ does not depend on $\sigma$,

$$
f(\boldsymbol{q})=\int d k \check{f}(k) e^{i k_{\mu} \boldsymbol{q}^{\mu}}
$$

is well defined, yet it does not belong to the spacetime algebra $\mathcal{E}=\mathcal{C}_{0}(\Sigma, \mathcal{K})$ since $f$ does not vanish at infinity as a function of $\sigma$. However, $f(\boldsymbol{q})$ is a multiplier of the algebra, in the sense that $f(\boldsymbol{q}) g(\boldsymbol{Q} ; \boldsymbol{q})$ is in $\mathcal{E}$ for every admissible symbol $g$. This can be understood also without knowing the abstract definition of multipliers algebra of a $C^{*}$-algebra ${ }^{17}$.

The functions of $\sigma$ alone also are natural multipliers: if $f=f(\sigma)$ is continuous and bounded and $g=g(\sigma ; x)$ is an admissible symbol, then $f(\sigma) g(\sigma ; x)$ is an admissible symbol and $f(\boldsymbol{Q}) g(\boldsymbol{Q} ; \boldsymbol{q})$ has an unambiguous meaning. Moreover, $f(\boldsymbol{Q})$ is a central multiplier: $f(\boldsymbol{Q}) g(\boldsymbol{Q} ; \boldsymbol{q})=g(\boldsymbol{Q} ; \boldsymbol{q}) f(\boldsymbol{Q})$; or, if we enjoy using exact terms, we may say that $f(\boldsymbol{Q})$ belongs to the centre $Z(M(\mathcal{E}))$ of the multipliers algebra $M(\mathcal{E})$ of $\mathcal{E}$. Note also that we have the natural identification,

$$
Z(M(\mathcal{E}))=\mathcal{C}_{b}(\Sigma)
$$

the algebra of bounded continuous functions of $\Sigma$.

The algebra $\mathcal{E}$ has no unit, because the only candidate would be, seen as a section, the constant function $\sigma \mapsto 1$; which does not vanish at infinity as a function of $\sigma$, and is not $L^{1}$ as a function

\footnotetext{
${ }^{17}$ This generalises a very simple situation: a bounded continuous function times a continuous function vanishing at infinity vanishes itself at infinity, hence $\mathcal{C}_{b}\left(\mathbb{R}^{4}\right)$ is the multipliers algebra of $\mathcal{C}_{0}\left(\mathbb{R}^{4}\right)$. It is the biggest algebra of functions with this property and thus it is a canonical object.
} 
of $x$ for any fixed $\sigma$. If we ask the same question while looking at the abstract $C^{*}$-algebra $\mathcal{E}=\mathcal{C}_{0}(\Sigma, \mathcal{K})$, we see that the only candidate to be the identity would be the constant function $\sigma \mapsto \mathbf{1}$; which does not vanish at infinity as a function of $\sigma$, and $\mathbf{1}$ is not compact. Of course, both candidates coincide with the unit of the multipliers algebra, up to natural identification.

Let us now consider the map

$$
f(\boldsymbol{Q} ; \boldsymbol{q}) \mapsto \int d x f(\boldsymbol{Q} ; x) \delta\left(x^{0}-t\right) .
$$

For every fixed $t$ this object depends on $\boldsymbol{Q}$ only, and is thus a central multiplier. The above map extends to a map from the whole $C^{*}$-algebra $\mathcal{E}$ to the central multipliers.

This map has a very interesting feature: it is positive, namely it sends positive elements into positive elements:

$$
\int d x f(\boldsymbol{Q} ; x)^{*} f(\boldsymbol{Q} ; x) \delta\left(x^{0}-t\right) \geqslant 0 .
$$

Since $f(\boldsymbol{Q} ; x)^{*} f(\boldsymbol{Q} ; x)=(\bar{f} \star f)(\boldsymbol{Q} ; x)$, the above can be written as

$$
\iint d x(\bar{f} \star f)(\boldsymbol{Q} ; x) \delta\left(x^{0}-t\right) \geqslant 0 .
$$

But central multipliers are identified with functions of $\sigma$, where positivity is just pointwise (in $\sigma$ ) positivity. Hence the above is equivalent to

$$
\int d x(\bar{f} \star f)(\sigma ; x) \delta\left(x^{0}-t\right) \geqslant 0, \quad \sigma \in \Sigma .
$$

In technical terms, the above map is a conditional expectation, since in addition it maps

$$
f(\boldsymbol{Q}) g(\boldsymbol{Q} ; \boldsymbol{q}) \mapsto f(\boldsymbol{Q}) \int d x g(\boldsymbol{Q} ; x) \delta\left(x^{0}-t\right) .
$$

In [7], the following suggestive notation was proposed for the above map:

$$
\int_{q^{0}=t} d^{3} q f(\boldsymbol{Q} ; \boldsymbol{q}):=\int d x f(\boldsymbol{Q} ; x) \delta\left(x^{0}-t\right) .
$$

Note that positivity with respect to the *-algebra is important, it essentially means compatibility with the uncertainty relations. For example, for every fixed $a \in \mathbb{R}^{4}$, the map $f(\boldsymbol{Q} ; \boldsymbol{q}) \mapsto$ $f(\boldsymbol{Q} ; a)$ (evaluation at a point) is not positive, since there's no reason why $(\bar{f} \star f)(\sigma, a)$ should define a positive function of $\sigma$; on the contrary, it cannot be positive, for otherwise it would allow to construct (by further integrating over $\sigma$ ) an admissible localisation state which would violate the uncertainty relations. The physical interpretation of positivity of the conditional expectation (4.6) is that localisation at sharp time can be compensated by complete space delocalisation.

The other important notion is that of derivative. Since we have the action of translations, we may define $\partial f(\boldsymbol{q}) / \partial \boldsymbol{q}^{\mu}$ by

$$
f(\boldsymbol{q}+\varepsilon)=f(\boldsymbol{q})+\varepsilon^{\mu} \frac{\partial f(\boldsymbol{q})}{d \boldsymbol{q}^{\mu}}+o(\varepsilon) .
$$

A short computation shows that it is precisely the same as the quantisation of $\partial_{\mu} f$ :

$$
\frac{\partial f(\boldsymbol{q})}{d \boldsymbol{q}^{\mu}}=\left(\partial_{\mu} f\right)(\boldsymbol{q}) .
$$


Finally, a comment on the states. Let $\omega$ be a state on $\mathcal{E}$, namely a liner functional on $\mathcal{E}$ which is positive and normalised. Examples can be obtained by thinking of $f(\boldsymbol{Q} ; \boldsymbol{q})$ as of an operator: vector states $^{18}$

$$
f(\boldsymbol{Q} ; \boldsymbol{q}) \mapsto \frac{\langle\xi|f(\boldsymbol{Q} ; \boldsymbol{q})| \xi\rangle}{\langle\xi \mid \xi\rangle}
$$

and more general states of the form

$$
f(\boldsymbol{Q} ; \boldsymbol{q}) \mapsto \operatorname{Tr}(\rho f(\boldsymbol{Q} ; \boldsymbol{q})),
$$

where $\rho$ is a positive operator with trace 1. A (regular) state can be extended to the multipliers. Since the central multipliers are an algebra of functions, then for any (regular) state $\omega$ there is a measure $\mu_{\omega}$ on $\Sigma$ such that, for any central multiplier $f(\boldsymbol{Q})$,

$$
\omega(f(\boldsymbol{Q}))=\int d \mu_{\omega}(\sigma) f(\sigma) .
$$

A state is said with optimal localisation if it minimises

$$
\sum_{\mu} \Delta_{\omega}\left(\boldsymbol{q}^{\mu}\right)^{2}
$$

This quantity is not Lorentz invariant, hence this definition depends on the observer, and the pull back of such a state to a different Lorentz frame does not have optimal localisation, in general. Such states exist and are essentially given in terms of the quantum mechanical coherent states. It can be shown that, if $\omega$ has optimal localisation, then the associated measure $\mu_{\omega}$ is supported by the orbit $\Sigma_{1} \subset \Sigma$ of the standard simplectic matrix $S$ under the space-orthogonal subgroup of $\mathscr{L}$. See [7] for more details.

\subsection{Classical limit}

It is clear that, when $\lambda_{P}$ goes to zero, then $f \star g$ goes to the pointwise product in the $x$ variable, fibrewise in the variable $\sigma$ :

$$
(f \star g)(\sigma ; x) \underset{\lambda_{P} \rightarrow 0}{\longrightarrow} f(\sigma ; x) g(\sigma ; x) .
$$

The classical limit of the DFR model, then, is the same as the large dilations limit, and is $\mathbb{R}^{4} \times \Sigma$, the usual Minkowski spacetime, times a manifold carrying extra dimensions. This comment may be made rigorous using Rieffel's theory of deformations [14].

The manifold $\Sigma$ has two connected components $\Sigma_{ \pm}$, each of which is homeomorphic to the tangent space of the 2 -sphere, so that there are four extra dimensions ${ }^{19}$. Hence the classical limit can be described as $\mathbb{R}^{4} \times T S^{2} \times\{ \pm 1\}$.

An unpleasant fact is that the extra dimensions are not compact, and thus in principle could be observed at the macroscopic scale. One way out would be to restrict the representation to the states which, in the large scale limit, will become sharp points; namely the states with optimal localisation. We recall that states with optimal localisation, restricted to the centre, are supported by $\Sigma_{1}$, which, under the homeomorphism $\Sigma_{ \pm} \rightarrow T S^{2}$, is mapped precisely to the 2-sphere. Hence in this case, the classical limit would be $\mathbb{R}^{4} \times S^{2} \times\{ \pm 1\}$, with compact extra dimensions. Unfortunately, the price to pay is the breakdown of Lorentz covariance, so that different observers, connected by a Lorentz boost, would disagree on the classical limit.

\footnotetext{
${ }^{18}$ Since the defining representation is reducible, 'vector state' is not the same as 'pure state'.

${ }^{19}$ This can be seen directly, by observing that an antisymmetric 2-tensor has six independent entries, and there are two independent invariants defining the manifold $\Sigma$.
} 


\subsection{Towards quantum geometry}

At its most basic level, classical geometry describes the relations of families of points.

A first step towards a generalisation of classical geometry is to discuss independent events. Two quantum quantities are statistically independent if they commute; a natural way for constructing independent copies of the same observable $\boldsymbol{A}$ is by means of tensors product: $\boldsymbol{A}_{1}=\boldsymbol{A} \otimes \boldsymbol{I}, \boldsymbol{A}_{2}=\boldsymbol{I} \otimes \boldsymbol{A}$. We may follow this pattern for defining the quantum coordinates of $n$ independent events

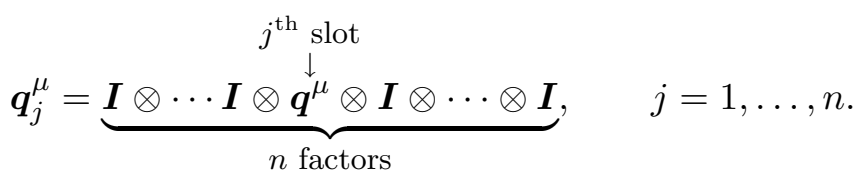

Then the quantisation of a function $f=f\left(x_{1}, \ldots, x_{n}\right)$ of many classical independent events is given by

$$
f\left(\boldsymbol{q}_{1}, \ldots, \boldsymbol{q}_{n}\right)=\int_{\mathbb{R}^{4 n}} d k_{1} \cdots d k_{n} \check{f}\left(k_{1}, \ldots, k_{n}\right) e^{i\left(k_{1} \boldsymbol{q}_{1}+\cdots k_{n} \boldsymbol{q}_{n}\right)},
$$

However, there are two natural notions of tensor product on the spacetime $C^{*}$-algebra. The usual one, which gives $\mathcal{E} \otimes \mathcal{E} \simeq \mathcal{C}_{0}(\Sigma \times \Sigma, \mathcal{K})$, namely functions of two independent $\sigma$ 's, and the fibrewise tensor product, according to which the tensor product of two sections is taken fibrewise. The abstract algebraic method for doing this is to understand $\otimes$ as a tensor product of $Z$-modules, where $Z$ is the centre of the multipliers algebra (see Section 4.4). Essentially, this amounts to identify the commutators of independent events:

$$
\left[\boldsymbol{q}_{j}^{\mu}, \boldsymbol{q}_{k}^{\nu}\right]=i \lambda_{P}^{2} \delta_{j k} \mathcal{Q}^{\mu \nu}
$$

where

$$
\mathcal{Q}^{\mu \nu}=\boldsymbol{Q}^{\mu \nu} \otimes \boldsymbol{I} \otimes \cdots \otimes \boldsymbol{I}=\boldsymbol{I} \otimes \boldsymbol{Q}^{\mu \nu} \otimes \cdots \otimes \boldsymbol{I}=\cdots=\boldsymbol{I} \otimes \boldsymbol{I} \otimes \cdots \otimes \boldsymbol{Q}^{\mu \nu} .
$$

That this is the natural notion of tensor product is confirmed by the remark that the separations between two independent events has the same commutation relations of the basic coordinates, up to a factor: if we set

$$
\delta_{j k} \boldsymbol{q}=\boldsymbol{q}_{j}-\boldsymbol{q}_{k},
$$

we find

$$
\left[\delta_{j k} \boldsymbol{q}^{\mu}, \delta_{j k} \boldsymbol{q}^{\nu}\right]=2 \lambda_{P}^{2} \mathcal{Q}^{\mu \nu}, \quad j \neq k,
$$

where the right hand side does not depend on $j, k[19,10]$. Now the square Euclidean distance $\sum_{\mu}\left(\delta_{j k} \boldsymbol{q}^{\mu}\right)^{2}$ is bounded below by ${ }^{20} 4 \lambda_{P}^{2}$; hence there is a natural minimal Euclidean distance between independent events [20].

Another pleasant feature which follows from adopting the fibrewise tensor product is that the barycentric coordinates and the separations are independent. Indeed, setting

$$
\overline{\boldsymbol{q}}=\frac{1}{n}\left(\boldsymbol{q}_{1}+\cdots+\boldsymbol{q}_{n}\right)
$$

\footnotetext{
${ }^{20}$ This can be seen directly in any state which is determined on $\mathcal{Q}$ with expectation $\sigma \in \Sigma_{1}$; in this case, we only see the irreducible component corresponding to the special representation described in Section 3.5, in which case the square Euclidean distance of two independent events is twice the Hamiltonian of a four dimensional Harmonic oscillator. See [7] for a proof that this is optimal.
} 
we find

$$
\left[\overline{\boldsymbol{q}}^{\mu}, \delta_{i j} \boldsymbol{q}^{\nu}\right]=0 .
$$

This is true in a strong sense, so that $\overline{\boldsymbol{q}}$ can be understood to live in a different tensor factor than the separations (up to an isomorphism). Then it is possible to set all the $\delta_{i j} \boldsymbol{q}^{\nu}$ to their minimum value, thus leaving a function of $\overline{\boldsymbol{q}}$ alone, with a hidden $\sigma$ dependence consisting of restriction to $\Sigma_{1}$ (due to the fact that the Euclidean distance is not covariant under Lorentz boosts).

What we obtain in this way is a quantum analogue of the restriction

$$
f\left(x_{1}, \ldots, x_{n}\right) \Upsilon_{x_{1}=x_{2}=\cdots=x}
$$

to the diagonal of a function of many events $[19,10]$.

In the framework of Dubois-Violette universal calculus [21], we may define $d \boldsymbol{q}=\boldsymbol{q} \otimes \boldsymbol{I}-\boldsymbol{I} \otimes \boldsymbol{q}=$ $\boldsymbol{q}_{2}-\boldsymbol{q}_{1}$, where, as everywhere in this section, we understand $\otimes$ "fibrewise" (or in the sense of $Z$-modules). A natural definition of 4 -volume is then

$$
\boldsymbol{V}=d \boldsymbol{q} \wedge d \boldsymbol{q} \wedge d \boldsymbol{q} \wedge d \boldsymbol{q}=\epsilon_{\mu \nu \rho \sigma}\left(\boldsymbol{q}_{2}-\boldsymbol{q}_{1}\right)^{\mu}\left(\boldsymbol{q}_{3}-\boldsymbol{q}_{2}\right)^{\nu}\left(\boldsymbol{q}_{4}-\boldsymbol{q}_{3}\right)^{\rho}\left(\boldsymbol{q}_{5}-\boldsymbol{q}_{4}\right)^{\sigma}
$$

(which is intuitively reminiscent of the volume of a hypercube), and analogously for 3-volumes and area operators. The spectra of all these operators can be computed; in particular the spectrum of $\boldsymbol{V}$ does not contain 0 , and stays at a finite distance $\sim \lambda_{P}^{4}$ from it [20].

\subsection{Geometry of $\Sigma$; dilation covariance}

Given a set of pairwise commuting operators $\boldsymbol{Q}^{\mu \nu}$ fulfilling $\boldsymbol{Q}^{\mu \nu}=-\boldsymbol{Q}^{\nu \mu}$, we may consider them as the entries of an antisymmetric matrix with operator entries

$$
\boldsymbol{Q}=\left(\begin{array}{llll}
\boldsymbol{Q}^{00} & \boldsymbol{Q}^{01} & \boldsymbol{Q}^{02} & \boldsymbol{Q}^{03} \\
\boldsymbol{Q}^{10} & \boldsymbol{Q}^{11} & \boldsymbol{Q}^{12} & \boldsymbol{Q}^{13} \\
\boldsymbol{Q}^{20} & \boldsymbol{Q}^{21} & \boldsymbol{Q}^{22} & \boldsymbol{Q}^{23} \\
\boldsymbol{Q}^{30} & \boldsymbol{Q}^{31} & \boldsymbol{Q}^{32} & \boldsymbol{Q}^{33}
\end{array}\right)=\left(\begin{array}{cccc}
\mathbf{0} & \boldsymbol{e}_{1} & \boldsymbol{e}_{2} & \boldsymbol{e}_{3} \\
-\boldsymbol{e}_{1} & \mathbf{0} & \boldsymbol{m}_{3} & -\boldsymbol{m}_{2} \\
-\boldsymbol{e}_{2} & -\boldsymbol{m}_{3} & \mathbf{0} & \boldsymbol{m}_{1} \\
-\boldsymbol{e}_{3} & \boldsymbol{m}_{2} & -\boldsymbol{m}_{1} & \mathbf{0}
\end{array}\right)
$$

where, following [7], we introduced the notation ${ }^{21} \boldsymbol{e}_{j}=\boldsymbol{Q}^{0 j}$ and $\boldsymbol{m}_{i}=\boldsymbol{Q}^{j k}((i, j, k)$ a cyclic permutation of $(1,2,3))$. The (pseudo-)vectors $\overrightarrow{\boldsymbol{e}}, \overrightarrow{\boldsymbol{m}}$ are called the electric and magnetic parts of $\boldsymbol{Q}$, in formal analogy with electromagnetism (no physical meaning attached to this terminology). Note that no meaning is attached to the position of the indices of $\overrightarrow{\boldsymbol{e}}, \overrightarrow{\boldsymbol{m}}$.

Correspondingly, we may represent the matrix $\boldsymbol{Q}$ as a pair $(\overrightarrow{\boldsymbol{e}}, \overrightarrow{\boldsymbol{m}})$ of vectors with operator entries. Now, given a matrix $\sigma \in \Sigma$, we may represent it as a pair $(\vec{e}, \vec{m})$ of vectors with real entries; we recall that, for the DFR model, $\operatorname{spec}\left(\boldsymbol{Q}^{\mu \nu}\right)=\left\{\sigma^{\mu \nu}: \sigma \in \Sigma\right\}$; under the above correspondence, $\operatorname{spec}\left(\boldsymbol{e}_{j}\right)=\left\{\sigma^{0 j}: \sigma \in \Sigma\right\}=\left\{e_{j}:(\vec{e}, \vec{m}) \in \Sigma\right\}$ and $\operatorname{spec}\left(\boldsymbol{m}_{j}\right)=\left\{m_{j}:(\vec{e}, \vec{m}) \in \Sigma\right\}$.

For the DFR model, $\Sigma$ is the orbit of the matrix $\left(\vec{e}_{3}, \vec{e}_{3}\right)$, where $\vec{e}_{3}=(0,0,1)$ is the third vector in the canonical basis of $\mathbb{R}^{3}$.

The advantage of this notation is that the two invariants take a very simple form:

$$
\boldsymbol{Q}_{\mu \nu} \boldsymbol{Q}^{\mu \nu}=2\left(|\overrightarrow{\boldsymbol{m}}|^{2}-|\overrightarrow{\boldsymbol{e}}|^{2}\right), \quad \boldsymbol{Q}_{\mu \nu}(* \boldsymbol{Q})^{\mu \nu}=4(\overrightarrow{\boldsymbol{m}} \cdot \overrightarrow{\boldsymbol{e}}) .
$$

It follows that

$$
\Sigma=\{(\vec{e}, \vec{m}):|\vec{e}|=|\vec{m}|, \vec{e} \cdot \vec{m}= \pm 1\} .
$$

\footnotetext{
${ }^{21}$ There are slight differences with formulas in [7], since here we define $\overrightarrow{\boldsymbol{e}}, \overrightarrow{\boldsymbol{m}}$ with respect to indices in upper position.
} 
We observed at the end of Section 4.3 that the Planck scale is given by the spectrum of the operator $\left[\boldsymbol{q}^{0}, \boldsymbol{q}^{1}, \boldsymbol{q}^{2}, \boldsymbol{q}^{3}\right]$. There is however a natural way to construct a model with $\left[\boldsymbol{q}^{0}, \boldsymbol{q}^{1}, \boldsymbol{q}^{2}, \boldsymbol{q}^{3}\right]=0$, where there is no characteristic length. This model is covariant under dilations, too (S. Doplicher, private conversation).

This can be obtained by replacing $\Sigma$ with the orbit

$$
\Sigma_{\mathrm{conf}}=\{(\vec{e}, \vec{m}):|\vec{e}|=|\vec{m}|, \vec{e} \perp \vec{m}\}
$$

for example, $\left(\vec{e}_{1}, \vec{e}_{2}\right) \in \Sigma_{\text {conf }}$, which corresponds to the (non trivial) commutation relations

$$
\left[\boldsymbol{X}^{0}, \boldsymbol{X}^{1}\right]=\left[\boldsymbol{X}^{1}, \boldsymbol{X}^{3}\right]=i \boldsymbol{I}
$$

Then by Schur's lemma $\boldsymbol{X}^{2}$ and $\boldsymbol{X}^{0}+\boldsymbol{X}^{3}$ are multiples of the identity; there are $\infty^{2}$ equivalence classes of irreducible representations corresponding to the given choice of $\sigma=\left(\vec{e}_{1}, \vec{e}_{2}\right)$ :

$$
\left(\boldsymbol{X}^{0}, \boldsymbol{X}^{1}, \boldsymbol{X}^{2}, \boldsymbol{X}^{3}\right)=(\boldsymbol{Q}, \boldsymbol{P}, \alpha \boldsymbol{I}, \beta \boldsymbol{I}-\boldsymbol{Q}), \quad \alpha, \beta \in \mathbb{R} .
$$

Note that this is different than in the $\lambda_{P} \neq 0$ case, where to each $\sigma$ there corresponds a unique class of equivalence classes. However, essentially by the same methods of [7], it is possible to build up coordinates $\boldsymbol{q}^{\mu}$ with commutators $\boldsymbol{Q}^{\mu \nu}=-i\left[\boldsymbol{q}^{\mu}, \boldsymbol{q}^{\nu}\right]$ fulfilling

$$
\boldsymbol{Q}^{\mu \nu} \boldsymbol{Q}_{\mu \nu}=\boldsymbol{Q}^{\mu \nu}(* \boldsymbol{Q})_{\mu \nu}=0,
$$

and covariant under a unitary representation of the group generated by Poincaré transformations and dilations. Uncertainty relations and field theory will be analysed elsewhere.

\section{The "canonical" quantum spacetime}

The so called "canonical" quantum spacetime is defined by the relations

$$
\left[\boldsymbol{x}^{\mu}, \boldsymbol{x}^{\nu}\right]=i \theta^{\mu \nu} \boldsymbol{I} .
$$

According to the discussion of Section 3.5, the DFR argument grants the existence of at least one regular representation if there is a matrix $\Lambda$ such that $\theta^{\mu \nu}=\Lambda^{\mu}{ }_{\mu^{\prime}} \Lambda^{\nu}{ }_{\nu^{\prime}} S^{\mu^{\prime} \nu^{\prime}}$.

However, if no such $\Lambda$ is known to exist, everything is possible: there could be other inequivalent regular representations, or no representations at all. Even if $\Lambda$ exists, but is not invertible, there might be many other inequivalent representations which cannot be obtained in this way. In these cases, no general solution is known, and the representation theory of the given relations must be discussed case by case.

We consider two classes of examples in this section. In the next section, we discuss the representation theory of models where the time coordinate commutes with all the space coordinates $\left(\theta^{0 j}=0\right)$, and we show that the existence of representations depends on the particular choice of $\theta$ (and as a byproduct, we describe the method for classifying them all in the good cases).

In the rest of the section we will discuss the case where $\theta$ is a DFR matrix, in which case the representations always exist.

We will shortly comment on the conceptual implications of the breakdown of isotropy in the flat spacetime at Planck scale, and on the lack of motivations.

\subsection{Time/space commutative models and representations}

We consider the most general time/space commutative models where $\theta^{0 j}=0$, so that in particular $\left[\boldsymbol{x}^{0}, \boldsymbol{x}^{j}\right]=0$. 
Space/time commutative models have no direct physical motivations; they enjoy some fortune because they apparently remove an obstruction to the development of a unitary non local perturbative $S$-matrix. We shall later see that these obstructions only are due to an improper treatment of time ordering, the consequences of which are hidden by time/space commutativity.

For some $a, b, c \in \mathbb{R}$, we have

$$
\theta=\left(\begin{array}{cccc}
0 & 0 & 0 & 0 \\
0 & 0 & -a & -b \\
0 & a & 0 & -c \\
0 & b & c & 0
\end{array}\right)
$$

which means

$$
\left[\boldsymbol{x}^{0}, \boldsymbol{x}^{k}\right]=0, \quad\left[\boldsymbol{x}^{1}, \boldsymbol{x}^{2}\right]=-i a \boldsymbol{I}, \quad\left[\boldsymbol{x}^{1}, \boldsymbol{x}^{3}\right]=-i b \boldsymbol{I}, \quad\left[\boldsymbol{x}^{2}, \boldsymbol{x}^{3}\right]=-i c \boldsymbol{I},
$$

and where at least one of $a, b, c$ is not zero. We first seek for irreducible representations.

By Schur's lemma, $\boldsymbol{x}^{0}=q \boldsymbol{I}$ for some $q \in \mathbb{R}$. Now, according to the desired relations, the operator $\boldsymbol{T}=c \boldsymbol{x}^{1}-b \boldsymbol{x}^{2}+a \boldsymbol{x}^{3}$ should fulfil $\left[\boldsymbol{T}, \boldsymbol{x}^{\mu}\right]=0$ and thus, again by Schur's lemma, $\boldsymbol{T}$ should be a multiple of the identity. We assume, say, that $a \neq 0$; otherwise one may reason analogously (or permute the indices). Then it should be

$$
\boldsymbol{x}^{3}=\frac{1}{a}\left(b \boldsymbol{x}^{2}-c \boldsymbol{x}^{1}\right)+q^{\prime} \boldsymbol{I}
$$

for some $q^{\prime} \in \mathbb{R}$. In other words, the representation is irreducible if and only if $\boldsymbol{x}^{1}, \boldsymbol{x}^{2}$ are irreducible; but in view of this and their commutation relations, by von Neumann uniqueness we must have $\boldsymbol{x}^{1}=\boldsymbol{P}, \boldsymbol{x}^{2}=a \boldsymbol{Q}$ (up to equivalence) where $[\boldsymbol{P}, \boldsymbol{Q}]=-i \boldsymbol{I}$.

To sum up, the most general irreducible representation should have the form

$$
\boldsymbol{x}^{0}=q \boldsymbol{I}, \quad \boldsymbol{x}^{1}=\boldsymbol{P}, \quad \boldsymbol{x}^{2}=a \boldsymbol{Q}, \quad \boldsymbol{x}^{3}=\frac{b}{a} \boldsymbol{P}-\frac{c}{a} \boldsymbol{Q}+q^{\prime} \boldsymbol{I},
$$

for some $q, q^{\prime} \in \mathbb{R}$. But in this way, we find

$$
\left[\boldsymbol{x}^{1}, \boldsymbol{x}^{3}\right]=i \frac{c}{a} \boldsymbol{I}, \quad\left[\boldsymbol{x}^{2}, \boldsymbol{x}^{3}\right]=i b \boldsymbol{I}
$$

which are the desired relations if and only if $c=-a b, c=-b$ (with solutions $a=1, c=-b \in \mathbb{R}$ or $a \in \mathbb{R}, c=b=0$ ); this gives conditions for the existence of irreducible representations in the case $a \neq 0$. In other words, the relations (5.1) admit representations only for some choices of $\theta$. For the good choices, all irreducible representations are obtained in the above way.

Note that in the bad case where no representation exists, there is no Weyl quantisation, and there is no corresponding Weyl product. Of course the usual formula for the Weyl product still is meaningful and may well be taken as a standalone definition; but the resulting $*$-algebra would not admit $C^{*}$-completions.

On the other side, let us now assume that we are in the good case, and that representations exist. Hence we classified them all. Note however that, if we took any of such irreducible representations as our choice of the coordinates, we would find $e^{i k_{\mu} \boldsymbol{x}^{\mu}}=e^{i k^{0} q} e^{-i \vec{k} \cdot \overrightarrow{\boldsymbol{x}}}$ for the Weyl operators, which would give a very strange quantisation prescription, whose effect on the time variable would be evaluation at the fixed value $x^{0}=q$ :

$$
\int_{\mathbb{R}^{4}} d k e^{i k_{\mu} \boldsymbol{x}^{\mu}} \check{f}(k)=\frac{1}{(2 \pi)^{3}} \int_{\mathbb{R}^{3}} d \vec{x} \int_{\mathbb{R}^{3}} d \vec{k} f(q, \vec{x}) e^{-i \vec{k} \cdot(\overrightarrow{\boldsymbol{x}}-\vec{x})}
$$


Apart from describing a very strange classical limit with ... constant time $x^{0}=q$, it would be impossible to define an associated twisted product (which requires injectivity of the quantisation, not to be ill posed).

Hence we have no rights to make an arbitrary choice, and we must take into account all irreducible representations at once. By direct integral techniques ${ }^{22}$, we get the universal representation (in the case $a=b=-c=1$, say)

$$
\boldsymbol{\xi}^{0}=\boldsymbol{Q}_{2}, \quad \boldsymbol{\xi}^{1}=\boldsymbol{P}_{1}, \quad \boldsymbol{\xi}^{2}=\boldsymbol{Q}_{1}, \quad \boldsymbol{\xi}^{3}=\boldsymbol{P}_{1}+\boldsymbol{Q}_{1}+\boldsymbol{Q}_{3},
$$

where $\boldsymbol{Q}_{1}, \boldsymbol{Q}_{2}, \boldsymbol{Q}_{3}$, are the Schrödinger position operators for a particle in the 3 -space, and $\boldsymbol{P}_{1}$ is the Schrödinger momentum fulfilling $\left[\boldsymbol{P}_{1}, \boldsymbol{Q}_{k}\right]=-i \delta_{1 k} \boldsymbol{I}$. By construction (or a direct check), they fulfil

$$
\left[\boldsymbol{\xi}^{\mu}, \boldsymbol{\xi}^{\nu}\right]=i \theta^{\mu \nu} \boldsymbol{I}
$$

as required. The Weyl quantisation is injective, the twisted product is well defined, and there is a unique $C^{*}$-completion of the resulting algebra, which is $\mathcal{C}_{0}\left(\mathbb{R}^{2}\right) \otimes \mathcal{K}$.

\section{2 "Canonical" quantum spacetime and DFR model}

From now on, we stick to the case of DFR matrices, where $\theta=\lambda_{P}^{2} \sigma$ for $\sigma \in \Sigma$. In this case the "canonical" quantum spacetime is nothing but one single fibre over the DFR $C^{*}$-bundle $\mathcal{E}=\mathcal{C}_{0}(\Sigma, \mathcal{K})$, precisely the fibre over the chosen $\sigma$. Abstractly, the fibre is the same for all $\sigma \mathrm{s}$, hence the choice of a particular $\sigma$ only entails a particular choice of the Weyl quantisation prescription, and the corresponding (reduced) twisted product. Let us write

$$
\boldsymbol{q}_{(\sigma)}^{\mu}=\lambda_{P} \Lambda_{\nu}^{\mu} \boldsymbol{X}^{\mu}
$$

where the $\boldsymbol{X}^{\mu}$ 's are defined in Section 3.5 and $\Lambda$ is any ${ }^{23}$ Lorentz matrix fulfilling $\sigma^{\mu \nu}=$ $\Lambda^{\mu}{ }_{\mu^{\prime}} \Lambda^{\nu}{ }_{\nu^{\prime}} S^{\mu^{\prime} \nu^{\prime}}$. Then by construction

$$
\left[\boldsymbol{q}_{(\sigma)}^{\mu}, \boldsymbol{q}_{(\sigma)}^{\nu}\right]=i \lambda_{P}^{2} \sigma^{\mu \nu} \boldsymbol{I}
$$

and the quantisation over $\sigma$ is defined by

$$
f\left(\boldsymbol{q}_{(\sigma)}\right)=\int d k \check{f}(k) e^{i k_{\mu} \boldsymbol{q}_{(\sigma)}^{\mu}} ;
$$

it maps admissible symbols into compact operators on $L^{2}(\mathbb{R})$, and fulfils

$$
\left(f \star_{\sigma} g\right)\left(\boldsymbol{q}_{(\sigma)}\right)=f\left(\boldsymbol{q}_{(\sigma)}\right) g\left(\boldsymbol{q}_{(\sigma)}\right) .
$$

In mathematics, "canonical" is used to indicate something which is independent from arbitrary choices. Standing the arbitrariness of the choice of a particular $\sigma$, the terminology "canonical quantum spacetime" is totally unjustified.

As it stands, the spacetime quantisation associated with the reduction to a particular irreducible representation is clearly non covariant, and there is not much to say.

However, it is possible to use the DFR bundle of algebras to define a form covariant model, where however the relativity of observers is broken, and Wigner's approach to quantum symmetries is dismissed. It amounts to let $\theta$ transform as a tensor, and to attach to each reference

\footnotetext{
${ }^{22}$ Note that $\int_{\mathbb{R}}^{\oplus} d q \mathbb{C}=L^{2}(\mathbb{R})$, and $\int_{\mathbb{R}}^{\oplus} d q q \cdot=\boldsymbol{Q}$, the Schrödinger position.

${ }^{23}$ The matrix $\Lambda$ is defined up to elements of the stabiliser of $S$ in $\mathscr{L}$; however $\boldsymbol{q}_{(\sigma)}^{\mu}$ does not depend on this choice; it only depends on $\sigma$.
} 
frame $\mathcal{O}^{\prime}$ its own $\theta^{\prime}$, compatibly with Lorentz transformations of reference frames. This model had some fortune in the literature, and can be shown to be equivalent to yet another approach named "twisted covariance", which we shall discuss in Section 5.4.

The basic idea is to fix a specific $\theta$ with respect to a specific frame, and claim that the observer $\mathscr{O}$ in that particular frame "sees" the commutation relations (5.2). Let us call it the privileged observer.

Then an observer $\mathscr{O}^{\prime}$ in a frame related to the privileged frame by a Poincaré transformation $(\Lambda, a)$ will correspondingly "see" the commutation relations

$$
\left[\boldsymbol{q}_{\left(\sigma^{\prime}\right)}^{\mu}, \boldsymbol{q}_{\left(\sigma^{\prime}\right)}^{\nu}\right]=i \lambda_{P}^{2} \sigma^{\prime \mu \nu} \boldsymbol{I}
$$

The resulting formalism is then form-covariant; yet it is possible to classify the observers in an absolute way, according to the relations they "see". While in the DFR model all the observers (in the sense of special relativity) are equivalent, here there are infinitely many equivalence classes of observers, labeled by $\Sigma$; two observers are equivalent if and only if they are connected by a Poincaré transformation $(\Lambda, a)$ such that $\Lambda$ leaves $S$ unchanged $(=\Lambda$ is in the stabiliser of $S$ in $\mathscr{L})$.

\section{3 "Canonical quantum spacetime" and localisation states}

The situation of the end of the preceding section may be described as regarding the DFR bundle of algebras as a collection of algebras labeled by $\sigma$, together with a groupoid of automorphisms connecting pairs of algebras in that collection; the global algebraic structure carried by the fibrewise product of sections is dismissed.

An equivalent way of describing it is to retain the global algebraic structure, while restricting instead the class of admissible localisation states [22]. The best way to understand this argument is to think that there are the Gods, who can see the whole structure of the spacetime; and the poor human beings (the observers described by the theory), with limited capability of understanding the Universe.

Indeed, observers can test the algebra with the states at their disposal. Let us assume that, by a decision of the Gods, the privileged observer $\mathscr{O}$ only may test, in his own reference frame, the full DFR algebra with states such that each $\boldsymbol{Q}^{\mu \nu}$ is completely determined and has expectation $\sigma^{\mu \nu}$ :

$$
\omega\left(\boldsymbol{Q}^{\mu \nu}\right)=\sigma^{\mu \nu}, \quad \Delta_{\omega}\left(\boldsymbol{Q}^{\mu \nu}\right)=0 .
$$

We denote by $\mathscr{S}_{\mathscr{O}}$ this class of states. By definition, for any state $\omega \in \mathscr{S}_{\mathscr{O}}$ there is a state $w_{\omega}$ on $\mathcal{K}$ such that

$$
\omega(f(\boldsymbol{Q} ; \boldsymbol{q}))=w_{\omega}\left(f\left(\sigma ; \boldsymbol{q}_{(\sigma)}\right)\right),
$$

and all states in $\mathscr{S}_{\mathscr{O}}$ arise in this way. It follows that on one side

$$
\omega(f(\boldsymbol{Q} ; \boldsymbol{q}) g(\boldsymbol{Q} ; \boldsymbol{q}))=w_{\omega}\left(f\left(\sigma ; \boldsymbol{q}_{(\sigma)}\right) g\left(\sigma ; \boldsymbol{q}_{(\sigma)}\right)\right), \quad \omega \in \mathscr{S}_{\mathscr{O}}
$$

on the other,

$$
\omega((f \star g)(\boldsymbol{Q} ; \boldsymbol{q}))=w_{\omega}\left(\left(f(\sigma ; \cdot) \star_{\sigma} g(\sigma ; \cdot)\right)\left(\boldsymbol{q}_{(\sigma)}\right)\right), \quad \omega \in \mathscr{S}_{\mathscr{O}}
$$

In other words, being enabled to test the geometry only by means of states in $\mathscr{S}_{\mathscr{O}}$, the privileged observer will not recognise the full algebraic structure available to the Gods, and only will find the "canonical quantum spacetime" over $\sigma$; his symbol algebra will be $\mathcal{E}_{\sigma}$, his twisted product $\star_{\sigma}$, and so on and so forth. 
Let us now give a look at the situation of the primed observer $\mathscr{O}^{\prime}$, connected to $\mathscr{O}$ by the Poincaré transformation $(\Lambda, a)$. The states available to her are just those in the pull-back

$$
\mathscr{S}_{\mathscr{O}^{\prime}}=\left\{\omega\left(\boldsymbol{U}(\Lambda, a)^{-1} \cdot \boldsymbol{U}(\Lambda, a)\right): \omega \in \mathscr{S}_{\mathscr{O}},(\Lambda, a) \in \mathscr{P}\right\}
$$

of $\mathscr{S}_{\mathscr{O}}$. They are precisely the states such that each $\boldsymbol{Q}^{\mu \nu}$ is completely determined and has expectation $\sigma^{\prime \mu \nu}$. By repeating the discussion, his symbol algebra will be $\mathcal{E}_{\sigma^{\prime}}$, his twisted product $\star_{\sigma^{\prime}}$, and so on and so forth.

Hence we are in the following situation: there is a perfectly covariant model, the DFR model, where the equivalence of observers is fully enforced. Fixing a $\theta=\lambda_{P}^{2} \sigma$ in a particular frame is equivalent to put a non invariant constraint on that model, which amounts to dismiss a huge class of otherwise admissible localisation states by means of a non invariant selection criterion. The natural question is then: Why SHOuld We Dismiss ALL ThOse STATEs? This question was raised in $[22,23]$.

\subsection{Twisted covariance}

In this section, we discuss an apparently different approach to covariance, based on quantum deformations of the Lorentz group (in the spirit of quantum groups) [24, 25].

Following [22, 23], we will convince ourselves that this formalism is equivalent in spirit to work with the full DFR model, if we agree to dismiss a large classes of otherwise admissible localisation states (in the sense described in Section 5.3); "in spirit" meaning: up to the choice between Weyl quantisation and algebraic relations.

Again, we will take a bottom-up approach, and describe twisted covariance by adding further degrees of structure step by step, when necessary. This will help us to keep track of the various assumptions, and of the nature of the mathematical concepts.

\section{Twists}

We will start by considering a map $f \otimes g \mapsto f \star g$ of the form

$$
(f \star g)(x)=\int d a d b K_{2}(x, a, b) f(a) g(b), \quad x \in \mathbb{R}^{4} .
$$

The only specific initial assumption is that manipulations like

$$
\int d a \int d b=\int d b \int d a, \quad \lim \int=\int \lim
$$

are allowed (possibly in a weak sense). Functions will be assumed smooth at wish.

Under the above assumptions, for some $\xi_{2}$ such that $\xi_{2}(x, x)=x$, we may define

$$
\begin{aligned}
& F_{2}(x, y, a, b)=K_{2}\left(\xi_{2}(x, y), a, b\right), \\
& \left(\mathcal{F}_{2} f \otimes g\right)(x, y)=\int d a d b F_{2}(x, y, a, b) f(a) g(b) .
\end{aligned}
$$

With

$$
m_{2}: f \otimes g \mapsto f g, \quad \tilde{m}_{2}: f \otimes g \mapsto f \star g,
$$

it follows that

$$
\tilde{m}_{2}=m_{2} \circ \mathcal{F}_{2} .
$$

Note that we did not use associativity of $\star$, nor invertibility of $\mathcal{F}_{2}$. 


\section{Finite twisted covariance}

The only additional assumption now is invertibility of $\mathcal{F}_{2}$.

With $\mathscr{L}$ the Lorentz group, we define the actions of $\Lambda \in \mathscr{L}$

$$
(\gamma(\Lambda) f)(x)=f\left(\Lambda^{-1} x\right), \quad \gamma_{2}(\Lambda)=\gamma(\Lambda) \otimes \gamma(\Lambda)
$$

they are intertwined by the usual product:

$$
m_{2} \circ \gamma_{2}(\Lambda)=\gamma(\Lambda) \circ m_{2} .
$$

The twisted action of $\mathscr{L}$ is defined as

$$
\tilde{\gamma}_{2}(\Lambda)=\mathcal{F}_{2}^{-1} \circ \gamma_{2}(\Lambda) \circ \mathcal{F}_{2}
$$

it is conceived so that, by construction,

$$
\tilde{m}_{2} \circ \tilde{\gamma}_{2}(\Lambda)=\gamma(\Lambda) \circ \tilde{m}_{2}
$$

which may be regarded as a deformation of (5.3). Note that in (5.4) the action $\gamma(\Lambda)$ on functions of one variable is unchanged. Equation (5.3) expresses usual covariance of the pointwise product; (5.4) is called twisted covariance of the twisted product.

We will need the following commutation relation: let $\mathcal{F}_{2}^{(\Lambda)}$ be defined by

$$
\gamma_{2}(\Lambda) \circ \mathcal{F}_{2}=\mathcal{F}_{2}^{(\Lambda)} \circ \gamma_{2}(\Lambda)
$$

then of course

$$
\left(\mathcal{F}_{2}^{(\Lambda)} f \otimes g\right)(x, y)=\int d a d b F_{2}(\Lambda x, \Lambda y, \Lambda a, \Lambda b) f(a) g(b) .
$$

\section{Infinitesimal twisted covariance}

Here too, we only assume invertibility of $\mathcal{F}_{2}$. Let us define $X^{\mu}$ by setting $\left(X^{\mu} f\right)(x)=x^{\mu} f(x)$; with $\omega \in \operatorname{Lie}(\mathscr{L})$, we have the action

$$
\omega \triangleright=\omega^{\mu}{ }_{\nu} X^{\nu} \partial_{\mu}
$$

so that

$$
\gamma\left(e^{t \omega}\right) f=f-t \omega \triangleright f+o(t)
$$

Let $\Delta$ be the coproduct for the usual (undeformed) coalgebra structure of the the universal enveloping Lie algebra $U(\operatorname{Lie}(\mathscr{L}))$ of the Lie algebra of $\operatorname{Lie}(\mathscr{L})$ of the Lorentz group. With

$$
\Delta(\omega)=\omega \otimes 1+1 \otimes \omega
$$

we have the action

$$
\Delta(\omega) \triangleright=(\omega \triangleright) \otimes I+I \otimes(\omega \triangleright)
$$

so that

$$
\gamma_{2}\left(e^{t \omega}\right) f \otimes g=f \otimes g-t \Delta(\omega) \triangleright f \otimes g+o(t) .
$$


Moreover,

$$
\tilde{\gamma}_{2}\left(e^{t \omega}\right) f \otimes g=f \otimes g-t \widetilde{\Delta(\omega) \triangleright} f \otimes g+o(t),
$$

where

$$
\widetilde{\Delta(\omega) \triangleright}=\mathcal{F}_{2}^{-1} \circ(\Delta(\omega) \triangleright) \circ \mathcal{F}_{2} .
$$

Note that the above defines a twisted action of the undeformed coproduct.

By (5.5),

$$
\tilde{\gamma}_{2}(\Lambda)=\mathcal{F}_{2}^{-1} \circ \mathcal{F}^{(\Lambda)} \circ \gamma_{2}(\Lambda)
$$

Hence

$$
\widetilde{\Delta(\omega) \triangleright}=\left.\frac{d}{d t}\left(\mathcal{F}^{-1} \circ \mathcal{F}^{\left(e^{-t \omega}\right)} \circ \gamma_{2}\left(e^{-t \omega}\right)\right)\right|_{t=0}=(\Delta(\omega) \triangleright)+\left.\mathcal{F}^{-1} \frac{d}{d t} \mathcal{F}^{\left(e^{-t \omega}\right)}\right|_{t=0} .
$$

\section{Weak coassociativity}

Besides invertibility of $\mathcal{F}_{2}$, now we also assume that $\star$ is associative, namely $(f \star g) \star h=f \star(g \star h)$. With $K_{3}$ defined by

$$
(f \star g \star h)(x)=\int d a d b d c K_{3}(x ; a, b, c) f(a) g(b) h(c),
$$

associativity implies that

$$
K_{3}(x ; a, b, c)=\int d y K_{2}(x ; y, c) K_{2}(y ; a, b)=\int d y K_{2}(x ; a, y) K_{2}(y ; b, c)
$$

within integrals. We may now reproduce all the steps: for some $\xi_{3}$ such that $\xi_{3}(x, x, x)=x$, define

$$
\left(\mathcal{F}_{3} f \otimes g \otimes h\right)(x, y, z)=\int d a d b d c K_{3}\left(\xi_{3}(x, y, z) ; a, b, c\right) f(a) g(b) h(c) ;
$$

with $m_{3} f \otimes g \otimes h=f g h$, set

$$
\tilde{m}_{3}(f \otimes g \otimes h)=f \star g \star h=m_{3} \circ \mathcal{F}_{3} f \otimes g \otimes h ;
$$

if $\mathcal{F}_{3}$ is invertible, set

$$
\begin{aligned}
& \gamma_{3}(\Lambda)=\gamma(\Lambda) \otimes \gamma(\Lambda) \otimes \gamma(\Lambda), \\
& \tilde{\gamma}_{3}=\mathcal{F}_{3}^{-1} \circ \gamma_{3} \circ \mathcal{F}_{3}=\mathcal{F}_{3}^{-1} \circ \mathcal{F}_{3}^{(\Lambda)} \circ \gamma_{3}
\end{aligned}
$$

it follows that

$$
\tilde{m}_{3} \circ \tilde{\gamma}_{3}(\Lambda)=\gamma(\Lambda) \circ \tilde{m}_{3} .
$$

As for infinitesimal transformations,

$$
\tilde{\gamma}_{3}\left(e^{t \omega}\right) f \otimes g \otimes h=\left(\mathrm{id}_{3}+\widetilde{\Delta_{3}(\omega)} \triangleright+o(t)\right) f \otimes g \otimes h,
$$

where

$$
\widetilde{\Delta_{3}(\omega)} \triangleright=\mathcal{F}_{3}^{-1} \circ \Delta_{3}(\omega) \circ \mathcal{F}_{3}=\Delta_{3}(\omega) \triangleright+\left.\mathcal{F}_{3} \circ \frac{d}{d t} \mathcal{F}_{3}^{\left(e^{-t \omega}\right)}\right|_{t=0} .
$$


Above $\Delta_{3}(\omega)$ is obtain by iteration of the undeformed, coassociative coproduct:

$$
\Delta_{3}(\omega)=(\Delta(\omega) \otimes 1) \circ \Delta(\omega)=(1 \otimes \Delta(\omega)) \circ \Delta(\omega) .
$$

Now, if we choose $\xi$ symmetric, namely

$$
\xi\left(x_{1}, x_{2}\right)=\xi\left(x_{2}, x_{1}\right),
$$

then we have weak twisted coassociativity, namely coassociativity of the twisted action:

$$
\widetilde{\Delta_{3}(\omega) \triangleright}=((\widetilde{\Delta(\omega) \triangleright}) \otimes I) \circ \widetilde{\Delta(\omega) \triangleright}=(I \otimes(\widetilde{\Delta(\omega) \triangleright})) \widetilde{\Delta(\omega) \triangleright} .
$$

\section{Strict twisted covariance}

The above steps where performed under rather mild assumptions. Maybe the most restrictive assumption is the invertibility of the twist operators; in the cases where they turn out to be invertible, it only requires some care with the analytic aspects to obtain weakly coassociative twisted covariance for a sufficiently regular model based on the Weyl quantisation of some set of relations.

However, in the above setting the Hopf algebra $U(\operatorname{Lie}(\mathscr{L}))$ associated with the Lie group $\mathscr{L}$ remains in the background: the coproduct is the usual one, twists only affect its action.

We now make the final step: the additional bit of structure is to assume that $\mathcal{F}_{2}=F_{2} \triangleright$ for some (invertible) $F_{2} \in U(\operatorname{Lie}(\mathscr{L})$ ) which fulfils some requirements (enforcing twisted coassociativity). In that case we can drop the symbol $\triangleright$ and define a new coproduct

$$
\tilde{\Delta}(\cdot)=F_{2}^{-1} \Delta(\cdot) F_{2} .
$$

Hence we have the following situation. Weakly twisted Poincaré covariance is possible both for the "canonical" quantum spacetime $[23,22]$ (see also next paragraph) and the $\kappa$-Minkowski spacetime $[12,26]$. In the first case, we can make the last step to strict twisted covariance [24, 25]. In the second case, strict twisted Poincaré covariance is not possible in the case of $\kappa$-Minkowski spacetime because of the obstructions to the existence of a suitable $F_{2}$ (although this obstruction can be circumvented by adding the dilation group, see [27]).

\section{Canonical quantum spacetime and twisted covariance}

Let $\sigma \in \Sigma$ and $\boldsymbol{q}_{(\sigma)}^{\mu}, \star_{\sigma}$ be the corresponding irreducible coordinates and star product.

We may restrict ourselves to the Schwartz functions, which are dense in the algebra of symbols, and allow for most manipulations with integrals and limits.

We recall that, like the usual pointwise product becomes convolution under Fourier transform (where $\widehat{f g}=\hat{f} \times \hat{g}$ ), its deformation $\star_{\sigma}$ is related with the corresponding deformation $\tilde{\times}_{\sigma}$ of the convolution product again by Fourier theory:

$$
\widehat{f \star_{\sigma} g}=\hat{f} \tilde{\times}_{\sigma} \hat{g}
$$

where

$$
(\hat{f} \tilde{\times} \hat{g})(k)=\int d k f(h) g(k-h) e^{\frac{i}{2} h_{\mu} \sigma^{\mu \nu} k_{\nu}} .
$$

Let us introduce the notation

$$
c(\hat{f} \otimes \hat{g})=\hat{f} \times \hat{g}
$$


for the usual (undeformed) convolution; then we have

$$
c_{\sigma}(\hat{f} \otimes \hat{g})=\hat{f} \tilde{\times}_{\sigma} \hat{g}=\left(c \circ \mathcal{T}_{\sigma}\right) f \otimes g,
$$

where $\mathcal{T}_{\sigma}$ is simply the multiplication by $e^{(i / 2) \sigma^{\mu \nu} h_{\nu} \otimes k_{\mu}}$, and is evidently invertible. Hence an invertible $\mathcal{F}_{\sigma}$ exists, and we find

$$
f \star_{\sigma} g=m \circ \mathcal{F}_{\sigma}(f \otimes g) .
$$

Relation (5.5) now reads

$$
\gamma_{2}(\Lambda) \circ \mathcal{F}_{\sigma}=\mathcal{F}_{\sigma^{\prime}} \circ \gamma_{2}(\Lambda)
$$

where primes indicate usual Lorentz actions on scalars, 4-vectors, and functions. It follows that

$$
m_{\sigma} \circ \tilde{\gamma}^{(2)}(\Lambda)(f \otimes g)=m \circ \mathcal{F}_{\sigma} \circ \mathcal{F}_{\sigma}^{-1}\left(\mathcal{F}_{\sigma}^{-1} f \otimes g\right)^{\prime}=f^{\prime} \star_{\sigma^{\prime}} g^{\prime}=\left(f \star_{\sigma} g\right)^{\prime} .
$$

Hence the usual form-covariance (where $\sigma$ is treated as a tensor and the action of Lorentz transformations is the usual one, in the framework described in Section 5.3) is perfectly equivalent - as a formalism - to weakly coassociative twisted covariance, where

- $\sigma$ is treated as a constant,

- the Lorentz action on functions of one event is unmodified, and

- the Lorentz action on functions of two or more events is twisted.

In turn, we have seen that usual form-covariance applied to "canonical" quantum spacetime is equivalent to deal with the full DFR model, up to dismiss a huge class of otherwise admissible localisation states (Section 5.3).

\section{Quantum field theory}

\subsection{Local quantum fields}

It is wise to shortly revise the fundamental concepts underlying relativistic quantum field theory on classical spacetime ${ }^{24}$, for the purpose of identifying the concepts which will have to be modified, and the motivations for such modifications.

To formulate a relativistic quantum physics, it is necessary to find a way to establish a quantum version of Einstein locality (or causality, they are synonyms). The most natural way is to rely on the usual notion of statistical independence for quantum observables, namely commutativity. If two observables $\boldsymbol{A}, \boldsymbol{B}$ are localised at some events $x, y$ respectively, they must fulfil $[\boldsymbol{A}, \boldsymbol{B}]=0$ whenever $x-y$ is spacelike ${ }^{25}$.

The theory is said local if the above condition is fulfilled, and any possible observable is either localised, or in the algebra generated by the localised observables.

To sum up, in order to describe a relativistic theory, two new concepts (axioms?) are to be injected into quantum physics: 1) it must be meaningful to ask whether a certain observable is localised in any region of spacetime (ideally even at a sharp point) or not, and 2) Einstein causality must hold in the form of commutativity at spacelike distances (we confine ourselves to observable fields).

The other basic ingredient is a unitary representation $\boldsymbol{U}$ of the Poincaré group $\mathscr{P}$, implementing a symmetry of the system with the natural geometric interpretation; namely for any observable $\boldsymbol{A}$ localised at $x, \boldsymbol{U}(\Lambda, a) \boldsymbol{A} \boldsymbol{U}(\Lambda, a)^{-1}$ represents the same experimental procedure, either performed

\footnotetext{
${ }^{24}$ Sometimes this is referred to as "commutative quantum field theory" (CQFT), as opposed to NCQFT; this is a nonsensical terminology, see also footnote 1 at page 3 .

${ }^{25}$ We take the signature $(+---)$ for the Lorentz metric; hence $x$ is spacelike if $x_{\mu} x^{\mu}<0$.
} 
- in a different, equivalent laboratory as seen by the initial observer in her reference frame (active point of view), or

- in the original event, but seen by a different observer from his reference frame (passive point of view).

Note that, for the active point of view to be meaningful, the localisation of the device must belong to the specifications of the experimental setup associated with the observable.

The action of translations define the total energy-momentum $\boldsymbol{P}^{\mu}$ of the theory by $\boldsymbol{U}(I, a)=$ $e^{i a_{\mu} \boldsymbol{P}^{\mu}}$. In non relativistic quantum physics it is necessary to postulate that the energy is lower bounded (otherwise an infinite amount of energy could be extracted from the system). The relativistic version is that the joint spectral resolution $\boldsymbol{P}^{\mu}|p\rangle=p^{\mu}|p\rangle$ of $\boldsymbol{P}$ must fulfil the condition $p_{\mu} p^{\mu} \geqslant 0$. In the absence of spontaneously broken symmetries, we also require uniqueness of the lower energy state $|0\rangle$, called the vacuum.

Let $\boldsymbol{A}$ be an observable localised at the event $x_{0}$; then for any other event $x$ we may define a new observable $\phi(x)$ by setting

$$
\phi(x)=\boldsymbol{U}\left(I, x_{0}-x\right) \boldsymbol{A} \boldsymbol{U}\left(I, x_{0}-x\right)^{-1},
$$

where we take the active point of view. In other words, $\phi(x)$ describes the observable obtained by displacing the experimental setup of $\phi\left(x_{0}\right)=\boldsymbol{A}$ from its original event $x_{0}$ into the new event $x$. We are then naturally led to consider operator-valued "functions" of the spacetime. The active point of view forces us to require consistence with Einstein locality:

$$
[\phi(x), \phi(y)]=0, \quad x-y \text { spacelike. }
$$

Moreover, the above must be compatible with Poincaré symmetry:

$$
\boldsymbol{U}(\Lambda, a) \boldsymbol{\phi}(x) \boldsymbol{U}(\Lambda, a)^{-1}=\boldsymbol{\phi}\left(\Lambda^{-1}(x-a)\right) .
$$

A field fulfilling the above two conditions (Einstein locality and Poincaré covariance) is called a relativistic quantum field. If in a theory there are many independent observable fields ${ }^{26} \phi_{j}$, they must be relatively local:

$$
\left[\phi_{j}(x), \phi_{k}(y)\right]=0, \quad x-y \text { spacelike. }
$$

For several reasons (see [28] for a review) the above picture is too optimistic: quantum fields are too singular, and cannot be treated as ordinary functions; sharply localised fields " $\phi(x)$ " only are meaningful within integrals (namely: as distributions). While this fact should not necessarily be seen as disturbing (pointwise localised instruments would have been an idealisation in any case), it might be regarded as the very first manifestation that something might go wrong in the infinitely small.

In standard textbooks, this is usually taken care of at the technical level by assuming that quantum fields are elements of some class of operator valued distributions, defined on a suitable class of regular test functions. However in this way we miss a point which is very important to us: that in this game test functions do not play the rôle of elements of an algebra of functions; they should instead be thought of as linear functionals on that algebra!

So, let us elaborate this idea of "smearing". As we have seen, already on classical spacetime, the localisation of an observable must be made "fuzzy" by choosing a probability density $\rho(x) d x$, and smearing the field over that density:

$$
\phi(\rho)=\int \phi(x) \rho(x) d x .
$$

\footnotetext{
${ }^{26}$ Although for general reasons the theory might encompass unobservable fields with different commutation relations (related with global gauge symmetries) at this level this is irrelevant for our discussion.
} 
Since fields are too singular, these probability densities must be sufficiently regular (we usually take infinitely differentiable functions), and vanish at infinity sufficiently fast (usually one takes functions with compact support or faster than inverse polynomials). In particular, a probability measure of the form $\delta(x-a) d x$ (sharp localisation at $a \in \mathbb{R}^{4}$ ) is not available.

Since any complex function with the same properties (smoothness, fast decay) can be written as the linear combination of at most four regular probability densities, it is natural to dismiss the requirements of positivity and normalisation, and extend the fields to generic test functions by linearity: fields are usually defined as linear maps $\ell \mapsto \phi(\ell)$ from test functions (= linear functionals, not necessarily positive and/or normalised) to operators.

The notation $\ell$ for a test function is unconventional; the reason is that we wish to reserve the symbol $f$ for an element of the localisation algebra $\mathcal{C}_{0}\left(\mathbb{R}^{4}\right)$. If $\rho$ is a probability density, then

$$
f \mapsto \int f(x) \rho(x) d x
$$

is a well defined state on the localisation algebra $\mathcal{C}_{0}\left(\mathbb{R}^{4}\right)$. Moreover, any test function $\ell$ defines a continuous linear functional

$$
f \mapsto \int f(x) \ell(x) d x
$$

Hence, quantum fields $\phi(\cdot)$ should be thought of as maps from the (sufficiently regular) states of the localisation algebra to the operators on some Hilbert space; extended by linearity to the regular linear functionals.

Then an expression like $\int P((\phi(x)) \ell(x) d x$, where $P$ is some polynomial (e.g. a Wick polynomial), is to be interpreted in terms of pointwise (local) products of fields, smeared with a sufficiently regular linear functional $\ell$ on the localisation algebra.

\subsection{DFR quantisation of local quantum free fields}

Let $\boldsymbol{F}$ be a continuous function of $\mathbb{R}^{4}$, vanishing at infinity, and taking values in the operators on some Hilbert space (or in some $C^{*}$-algebra $\mathfrak{F}$ ). Then we may set

$$
\boldsymbol{F}(\boldsymbol{q})=\int d k e^{i k_{\mu} \boldsymbol{q}^{\mu}} \otimes \check{\boldsymbol{F}}(k)
$$

where

$$
\check{\boldsymbol{F}}(k)=\frac{1}{(2 \pi)^{4}} \int d x \boldsymbol{F}(x) e^{-i k_{\mu} x^{\mu}},
$$

is well defined (provided $\boldsymbol{F}, \check{\boldsymbol{F}}$ are in $L^{1}$ ).

We may apply the above to a function of the form $\boldsymbol{F}(x)=f(x) \boldsymbol{A}$, where $\boldsymbol{A}$ is some fixed operator (or element of $\mathfrak{F}$ ), and $f$ is a complex continuous function, and we get $\boldsymbol{F}(\boldsymbol{q})=f(\boldsymbol{q}) \otimes \boldsymbol{A}$. If we consider another function of the form $\boldsymbol{G}(x)=g(x) \boldsymbol{B}$, then we get $\boldsymbol{G}(\boldsymbol{q})=g(\boldsymbol{q}) \otimes \boldsymbol{B}$. Their product has two sources of non-commutativity: the original one, due to the fact that the functions take values in a non-commutative algebra; and the new one, due to the spacetime quantisation. In other words, already before spacetime quantisation the pointwise product was non-commutative: $(\boldsymbol{F} \boldsymbol{G})(x)=\boldsymbol{F}(x) \boldsymbol{G}(x) \neq \boldsymbol{G}(x) \boldsymbol{F}(x)=(\boldsymbol{G} \boldsymbol{F})(x)$, in general. Now spacetime quantisation in a sense "increases the non-commutativity".

The most general function $\boldsymbol{F}$ as above can be approximated as

$$
\boldsymbol{F}(x) \approx \sum_{j} f_{j}(x) \boldsymbol{A}_{j},
$$


so that

$$
\boldsymbol{F}(\boldsymbol{q}) \approx \sum_{j} f_{j}(\boldsymbol{q}) \otimes \boldsymbol{A}_{j}
$$

note that above $\boldsymbol{F}(\boldsymbol{q})$ is obtained by applying the DFR quantisation of ordinary symbols in the first tensor factor only, leaving the second tensor factor unmodified.

The exact mathematical meaning of (6.1) is that there is a canonical isomorphism between $\mathcal{C}_{0}\left(\mathbb{R}^{4}, \mathfrak{F}\right)$ and $\mathcal{C}_{0}\left(\mathbb{R}^{4}\right) \otimes \mathfrak{F}$, sending the function $x \mapsto f(x) A$ into the element $f \otimes A$. The meaning of (6.2) is that the quantisation of $\boldsymbol{F}(x)$ is obtained by combining the above mentioned isomorphism with the ordinary quantisation on the first tensor factor only.

The above comments embody the statement that the non-commutative replacement of $\mathcal{C}_{0}\left(\mathbb{R}^{4}, \mathfrak{F}\right)$ is $\mathcal{E} \otimes \mathfrak{F}$. But we have much more, we have a consistent quantisation recipe for the $\mathfrak{F}$-valued functions.

We may of course define a symbolic calculus, but, precisely as in the case of ordinary symbols, we must allow for more general symbols, namely $\mathfrak{F}$-valued functions $\boldsymbol{F}=\boldsymbol{F}(\sigma ; x)$ of $\Sigma \times \mathbb{R}^{4}$, and define their quantisation $\boldsymbol{F}(\boldsymbol{Q} ; \boldsymbol{q})$ consistently. This allows to define a twisted product by

$$
\boldsymbol{F}(\boldsymbol{Q} ; \boldsymbol{q}) \boldsymbol{G}(\boldsymbol{Q} ; \boldsymbol{q})=(\boldsymbol{F} \star \boldsymbol{G})(\boldsymbol{Q} ; \boldsymbol{q}),
$$

which again is fibrewise:

$$
(\boldsymbol{F} \star \boldsymbol{G})(\sigma ; x)=\boldsymbol{F}(\sigma ; \cdot) \star_{\sigma} \boldsymbol{F}(\sigma ; \cdot) .
$$

Now, a local free field $\phi$ is not continuous (it's a distribution), nor it takes values in a $C^{*}$ algebra (fields are unbounded operators, in general). However, precisely as in the classical case, they can be formally treated as ordinary functions to some extent (see any book on Wightman theory). Hence, following [7], we may give the formal recipe

$$
\boldsymbol{\phi}(\boldsymbol{q})=\int d k e^{i k_{\mu} \boldsymbol{q}^{\mu}} \otimes \check{\boldsymbol{\phi}}(k)
$$

for the quantisation of a given local free quantum field.

Note that, precisely in the same way as the label " $x$ " of a local quantum field $\phi(x)$ is not an observable, here the " $\boldsymbol{q}$ " of $\boldsymbol{\phi}(\boldsymbol{q})$ is not an observable!

Let $\omega$ be a localisation state on the quantum spacetime localisation algebra. We can apply it to the first tensor factor, and obtain

$$
\phi(\omega)=\int d k(\omega \otimes \mathrm{id})\left(e^{i k_{\mu} \boldsymbol{q}^{\mu}} \otimes \check{\boldsymbol{\phi}}(k)\right)=\int d x \boldsymbol{\phi}(x) \rho_{\omega}(x),
$$

where

$$
\rho_{\omega}(x)=\frac{1}{(2 \pi)^{4}} \int d k \omega\left(e^{i k_{\mu} \boldsymbol{q}^{\mu}}\right) e^{-i k_{\mu} \boldsymbol{x}^{\mu}} ;
$$

namely the initial local field $\phi(x)$ evaluated on a probability density on the classical spacetime. This gives us the definition of "sufficiently regular state $\omega$ ", as a state $\omega$ such that $\rho_{\omega}$ is an admissible test function for the initial field.

The question as to why we restricted ourselves to free fields has a simple and neat answer: no interacting field is known in four dimensions. 


\subsection{Perturbation theory - the Dyson series}

Local perturbation theory of local quantum fields on classical spacetime (in its more sophisticated version, namely renormalisation theory) has been very effective in giving extremely accurate predictions with wonderful experimental validation. Quite surprisingly, it works even if the interaction is not a "small" perturbation of the free dynamics in any reasonable sense.

As a first attempt to develop quantum field theory on quantum spacetime, we may expect that non local Planck scale corrections are "small" enough not to destroy the good behaviour of the local perturbation series. On the contrary, the fuzziness of quantum spacetime should have an intrinsic regularising effect on the ultraviolet (i.e. small scale) divergences.

We begin with an important remark. Let $\mathscr{H}\left(\phi, \partial_{\mu} \phi\right)$ be the usual (Wick ordered) Hamiltonian density of the free scalar (Klein-Gordon) field $\boldsymbol{\phi}$, with $\boldsymbol{H}_{0}=\int_{x_{0}=t} d \vec{x} \mathscr{H}\left(\boldsymbol{\phi}(x), \partial_{\mu} \boldsymbol{\phi}(x)\right.$ ) (as an operator on the Fock space; it does not depend on $t)$; then it was found in [7] that

$$
\int_{q_{0}=t} d^{3} q \mathscr{H}\left(\boldsymbol{\phi}(\boldsymbol{q}), \partial_{\mu} \phi(\boldsymbol{q})\right)=\boldsymbol{H}_{0} \quad(\text { as a constant function of } \sigma) \text {. }
$$

The exact meaning of $\int_{q_{0}=t} d^{3} q$ is explained in Section 4.4 (see in particular equation (4.6)).

The above remark suggests that the free theory remains consistent after spacetime quantisation. This is a rewarding confirmation, but not really a surprise. Indeed spacetime quantisation is a purely kinematical, fully covariant procedure, and the local free field is well defined and covariant as well.

The next step is to define, for each $t$,

$$
\boldsymbol{H}_{I}(\boldsymbol{Q} ; t)=\int_{q_{0}=t} d^{3} q: \phi(\boldsymbol{q})^{n}:
$$

which may be thought of as a non constant function of $\sigma$ (see Section 4.4). In symbolic language, we may rewrite the above as

$$
\boldsymbol{H}_{I}(\sigma ; t)=\int d x \delta\left(x^{0}-t\right):\left(\boldsymbol{\phi} \star_{\sigma} \cdots \star_{\sigma} \phi\right)(x): .
$$

Up to now, everything was perfectly satisfactory. However now we have the problem of the $\sigma$ dependence of $\boldsymbol{H}_{I}$. At some point of the story, it must be integrated out. The reason is that this is a model for particle scattering. Particles scattered far away are free and thus do not undergo high energy processes which could excite the quantum geometric background. They are to be described by the dear old free fields (as in- and out-fields).

There are many inequivalent possibilities. But they all are affected by the same problem: there is no Lorentz invariant measure on $\Sigma$. It's just a fact of life ${ }^{27}$. Hence whatever measure is chosen over $\Sigma$, this operations will break Lorentz covariance. We will come back to this problem later on.

The choice proposed in [7] is to take the rotation invariant measure $d \sigma$ on $\Sigma_{1} \subset \Sigma$, where $\Sigma_{1}$ was described at the end of Section 4.4. This choice is for the largest possible geometric symmetry. We get

$$
\boldsymbol{H}_{I}(t)=\int_{\Sigma_{1}} d \sigma \int d x \delta\left(x^{0}-t\right): \boldsymbol{\phi} \star_{\sigma} \cdots \star_{\sigma} \boldsymbol{\phi}:(x)
$$

as a non local replacement of the usual local perturbation of the Hamiltonian of the free field, in the interaction picture. This interaction is covariant under translations and space rotations, but not under Lorentz boosts.

\footnotetext{
${ }^{27}$ Technically it is a consequence of $\mathscr{L}^{+}$being not amenable.
} 
The opposite choice is to take for the measure on $\Sigma$ the Dirac measure concentrated on some special choice of $\sigma$, which gives the same result as if we would have restricted ourselves from the start to the "canonical quantum spacetime" corresponding to that choice of $\sigma$.

Note that, for any of the above choices, there is a suitable kernel $G_{t}$ such that

$$
\boldsymbol{H}_{I}(t)=\int_{\mathbb{R}^{4 n}} d a_{1} \cdots d a_{n} G_{t}\left(a_{1}, \ldots, a_{n}\right) \phi\left(a_{1}\right) \cdots \phi\left(a_{n}\right) .
$$

The scattering matrix ( $S$-matrix) is defined as the formal solution

$$
S=U(\infty,-\infty)
$$

of

$$
\frac{d}{d t} \boldsymbol{U}(t, s)=i \boldsymbol{H}_{I}(t) \boldsymbol{U}(t, s)
$$

fulfilling $\boldsymbol{U}(t, t)=\boldsymbol{I}$. It can be described in terms of Dyson's non-commutative modification of Picard's method:

$$
S=\sum_{n=0}^{\infty} \frac{1}{n !} \int d t_{1} \cdots d t_{n} T\left[\boldsymbol{H}_{I}\left(t_{1}\right), \ldots, \boldsymbol{H}_{I}\left(t_{n}\right)\right]
$$

the time ordered product is defined as

$$
T\left[\boldsymbol{H}_{I}\left(t_{1}\right), \ldots, \boldsymbol{H}_{I}\left(t_{n}\right)\right]=\boldsymbol{H}_{I}\left(t_{\pi(1)}\right) \cdots \boldsymbol{H}_{I}\left(t_{\pi(n)}\right),
$$

where $\pi$ is the permutation of $(1,2, \ldots, n)$ such that $t_{\pi(1)}>t_{\pi(2)}>\cdots>t_{\pi(n)}$.

The regularising effect of non locality on the Dyson series for this ansatz has not yet been fully investigated. However there are interesting partial results; in particular Bahns [29] found an ultraviolet finite $S$-matrix in a variant of this model for the $: \phi^{\star 3}$ : interaction, where the $\sigma$ variable is integrated out independently at any vertex of the resulting diagrams.

Other proposals, which go beyond the scope of this review, are possible for the generalisation of Wick product on quantum spacetime. Indeed, on classical spacetime $f g$ can be looked at in at least two ways: as a pointwise product $f \otimes g \mapsto f g$ in the algebra of functions, or as a limiting procedure $(f g)(x)=\lim _{y \rightarrow x} f(x) g(y)$. These two equivalent procedures have inequivalent generalisations to quantum spacetime: the first becomes $f \otimes g \mapsto f \star g$, while the second can be given a meaning by sending the differences $\boldsymbol{q}_{j}-\boldsymbol{q}_{k}$ to their minimum, compatibly with positivity (i.e. uncertainty relations), as described in Section 4.6. The first one is precisely the one used in [7], which we already have seen. The second is investigated in [10] and used to define another form of quantum Wick product; it results in a complete ultraviolet regularisation of the $S$-matrix. A third proposal is to give standalone definition of "local" subtractions of divergences for the product $\phi^{\star n}$; this gives yet another definition of quantum Wick product [30], whose behaviour in the Dyson series has not yet been investigated.

Not only there are many inequivalent generalisations of the Wick product. There also are inequivalent generalisations of the perturbative series. The Yang-Feldman equation, which is equivalent to the Dyson series on the classical spacetime, provides a different evolution series [31].

In all the approach described above, interactions sooner or later destroy Lorentz covariance. A striking example of this situation is provided by the approach based on the Yang-Feldman equation, which seems to be covariant at all steps; yet in the end it requires a $\sigma$-dependent mass renormalisation [30].

Since every more or less naive generalisation of local interactions seems to lead to a conflict with Lorentz covariance, it is reasonable to conjecture that the crucial point on which we should concentrate our efforts is to understand the fate of locality beyond non-commutativity. 


\subsection{Formal unitarity is not violated!}

In [32], unitarity violations of the $S$-matrix were found for the $\phi^{\star 4}$ theory, in the form of a failure of the optical theorem for a particular graph (the "fish").

However, it is clear that, if the interaction Hamiltonian is formally selfadjoint, the $S$-matrix is formally unitary, and no formal violations of unitarity can be expected. So, what went wrong?

There are two main sources of ambiguity: the Euclidean methods and the Moyal expansion. We first discuss the latter. To this end we consider the first order contribution to the two points $\tau$-function, arising upon insertion of the Dyson expansion into the Gell-Mann-Low formula ${ }^{28}$ :

$$
-\frac{1}{2} \int d t\left\langle 0\left|T\left[\phi(x), \phi(y), \boldsymbol{H}_{I}(t)\right]\right| 0\right\rangle,
$$

where the time ordering refers to the variables $x^{0}, y^{0}, t$.

For the sake of comparison we first recall a basic trick of the local theory, in the case of an interaction term of the form $H_{I}(t)=\int d^{4} a \delta\left(t-a^{0}\right): \phi^{n}(a):$. Plugging it into the above formula, and carrying first the integration over the variable $t$ we get

$$
-\frac{1}{2} \int_{\mathbb{R}^{4}} d a\left\langle 0\left|T\left[\phi(x), \phi(y),: \phi^{n}(a):\right]\right| 0\right\rangle,
$$

where now the time ordering refers to the variables $x^{0}, y^{0}, a^{0}$. Inadvertently, the time ordering has been shifted inside the integral which appear in the definition of $\boldsymbol{H}_{I}(t)$. The reason why this is possible is that the time parameter defining the interaction term is precisely the same as the time variable the Wick monomials are evaluated at; hence integration and time ordering commute.

The same trick cannot be done if instead we plug a non local interaction term of the form (6.4) into (6.5), which gives

$$
-\frac{1}{2} \int d t\left\langle 0\left|T\left[\phi(x), \phi(y), \int d a_{1} \cdots d a_{n} G_{t}\left(a_{1}, \ldots, a_{n}\right): \phi\left(a_{1}\right) \cdots \phi\left(a_{n}\right):\right]\right| 0\right\rangle,
$$

where the time ordering is relative to the variables $x^{0}, y^{0}, t$ and has no relations at all with the variables $a_{j}^{0}$. This comment was already available in [7], but was rediscovered much later: the correct time ordered prescription was then given the name of "Interaction Point Time Ordering Prescription" (IPTOP), which is a somewhat unfortunate terminology, since a basic feature on non commutative spacetimes is that the concept of point is not available any more.

Indeed, it was shown in [31] that, with the proper treatment of time ordering, the optical theorem for the "fish" graph holds true, and there is no violation of unitarity even if time and space do not commute. Indeed, this is only one single example; formal unitarity was already implied by the choice of using the Dyson series with the correct time ordering prescription.

It has been observed that, if one takes a time/space commutative "canonical quantum spacetime", than the violations of unitarity disappear, and this was taken as an indication that time/space non-commutativity was responsible for the unitarity violations. As we have seen, this is not the case. Formal unitarity violations were due to an improper treatment of the time ordering; the only effect of time/space commutativity is to cancel the effects of that error.

We observed in Section 3.4 that, in a sense, the Moyal expansion is non-commutative but local; non-locality being taken care of by analyticity. If one manipulates the Moyal expansion and formally treats the twist as if it were a true differential operator, then all the fields (external and internal vertices of Feynman diagrams) would be apparently evaluated at the same time and

\footnotetext{
${ }^{28}$ Here we omit the (infinite) normalisation $\frac{1}{\langle 0|S| 0\rangle}$; for an explicit proof that the cancellation of vacuum-vacuum components carries over also in the non local framework, see [33].
} 
we would be naturally led to bring the time ordering inside the integral, thus taking the wrong time ordering prescription.

Of course, this is not a proof that there exists a unitary theory; indeed, this would require having completed the renormalisation programme in the Minkowskian setting. On the contrary, this programme is quite underdeveloped, since it must be formulated in terms of operator fields, which is a formidable task already in the commutative case (this is precisely the reason of the fortune of Euclidean measure-theoretic methods on classical spacetime).

\subsection{Perturbation theory and diagrams}

The perturbative terms in the Gell-Mann-Low formula can be treated in a very economical way by means of Feynman diagrams, namely simple drawings representing complicate integrals, encoded in rules for drawing the diagrams. Unfortunately the situation in the non-commutative case is quite confusing.

The first set of rules for diagrams appeared in a paper by Thomas Filk [34]. Filk's approach was in the spirit of a standalone $S$-matrix theory, as described e.g. in $[35]^{29}$. So, his starting point was to consider the classical action, keep the quadratic (i.e. free) term unchanged (according to DFR analysis), and replace the pointwise product in the interaction term with the twisted product.

Unfortunately, the classical action arises in the Gell-Mann-Low formula precisely due to the trick of bringing the time ordering inside the integral (see any standard textbook on QFT); which is not allowed in the case of time/space non-commutativity. The Filk rules, then, can not be derived from the Hamiltonian evolution proposed in [7]. This is the first manifestation of a basic fact of life: it is not obvious that perturbative methods which are equivalent in the commutative case have equivalent non-commutative generalisations; usually they don't. In this case, the lack of unitarity could be regarded as a good reason to dismiss this approach.

This also explains why Filk rules turn out to fulfil unitarity in the case of time/space commutativity. Motivated by [31], the rules for the DFR Hamiltonian approach were developed in $[36,37]$ (see also the very clear [38]). In view of a remark of [39], the situation was further clarified in [33], to which we may refer for more details.

Additional sets of inequivalent rules for diagrams can be obtained in the Euclidean framework, discussed in the next section.

\subsection{Euclidean methods and UV/IR mixing}

There is a well known connection between local QFT and classical statistical mechanics. Indeed, due to translation covariance and the spectrum condition, vacuum expectations

$$
\mathscr{W}\left(x_{1}-x_{2}, x_{2}-x_{3}, \ldots, x_{n-1}-x_{n}\right)=\left\langle 0\left|\phi\left(x_{1}\right) \phi\left(x_{2}\right) \cdots \phi\left(x_{n}\right)\right| 0\right\rangle
$$

of local product of fields (Wightman functions) have analytic continuations to imaginary time (Wick rotation), and the resulting functions $S_{n}\left(\eta_{1}, \ldots, \eta_{n-1}\right)$ (the Schwinger functions) are totally symmetric; here $\eta_{j}^{0}=i\left(x_{j}-x_{j+1}\right)^{0}$. This suggests to think of them as of correlation functions of generalised Brownian motions, were the space of "paths" is a suitable space of distributions on $\mathbb{R}^{4}$, called Euclidean fields (or sometimes Euclidean field configurations). The probability measure tolling these "paths" is expressed in terms of the classical Euclidean action, which arises from the combination of the Wick rotation and of locality in time (the shift of time ordering inside the integral, discussed in Section 6.4). This allows for using methods from stochastic theory in the computation of Feynman diagrams; the idea is to finally switch back to

\footnotetext{
${ }^{29}$ It is well known that this approach was first sponsored by Werner Heisenberg; according to a private conversation reported by Rudolph Haag, however, Heisenberg then dismissed this view.
} 
real time to obtain physically observable quantities (inverse Wick rotation). This approach is called "Euclidean" because the Lorentzian square of a 4 -vector is positive definite at imaginary times, if the signature -+++ is taken.

Note that the Euclidean formulation is possible only at the level of expectation values (Wightman functions); no Euclidean operator field theory can be directly obtained by a Wick rotation, because of the sad fate of the time evolution operator $e^{i t \boldsymbol{H}}$, when computed at imaginary times.

Wightman functions enjoy a property called "Wightman positivity", which is equivalent to the positivity of transition probabilities. Thanks to locality and covariance, this property has a Euclidean counterpart, called "reflection positivity", or "Osterwalder-Schrader" positivity; it is necessary for a family of would-be Schwinger functions to be actually connected to some physical expectation values in Minkowski space (see e.g. the nice and readable textbook [40]). Note however that the correspondence between Wightman and OS axioms is not one-to-one; OS positivity is a joint consequence of Wightman positivity, locality and covariance.

It is evident that there are many potential sources of trouble in trying to extend this method to the non commutative case:

a) the original argument showing that the analytic continuation reaches imaginary times still may be valid in the case of time space commutativity, since it is based on the spectral condition (see d) here below);

b) since Lorentz covariance is broken, Schwinger functions cannot be expected to be symmetric, so their interpretation as correlation functions is lost, and the stochastic interpretation (if any) cannot be expected to be obtained by a simple deformation of the local partition function (which would give symmetric Schwinger functions);

c) no replacement for reflection positivity is available so far (note that it should reproduce usual reflection positivity in the large scale limit).

The above remarks are general; if in addition time does not commute with space,

d) twists blow up exponentially at imaginary times and destroy the analyticity argument for the Wick rotation based on the spectrum condition (see however [41]);

e) there is really no reason to expect any rôle for the classical Euclidean action, because of the time ordering issue.

Euclidean theory can (and is) of course be studied as a standalone $S$-matrix theory, but it is not clear which could be its physical content; without any guidance from first principles or experiments, there is little hope to guess appropriate Euclidean Feynman rules, giving a physical theory on Minkowski space up to some Wick rotation. Note that it also might be sensible to expect such a theory to have a local large scale limit.

Indeed, there are good indications that the current Euclidean approach is not related with the Minkowski formulation [41].

Since the standalone approach is rather fashionable, quite often authors do not state explicitly which is their perturbative setting. The joint consequences of Euclidean methods and exotic time ordering prescription are then quite difficult to disentangle. Writing diagrams in terms of the classical action would mean to take an exotic time ordering prescription on Minkowski space, if the Euclidean theory could be obtained by a naive Wick rotation (it can not).

An example where such a disentanglement would be particularly useful is in the issue of IR/UV mixing, namely the conjecture that infrared and ultraviolet divergences (usually decoupled in local QFT) get coupled in the non commutative case. This was first observed in the Euclidean setting in [42], where the following intuitive explanation is given: "Roughly, very small pulses instantaneously spread out very far upon interacting. In this manner very high energy processes have important long distance consequences." Were this true, then we could expect 
single particle collisions at Planck energy to have large detectable effects at astronomical distances, which would be quite surprising. The IR/UV mixing is now the strongest obstruction to renormalisation in the Euclidean setting; as we said, in the Minkowskian setting renormalisation is way underdeveloped, and it is not even crystal clear how to formulate it. As of today we do not know if IR/UV mixing (or similar features/pathologies) does arise as well in the Minkowskian setting (this claim could become outdated in the near future).

\section{Acknowledgements}

I am indebted with Dorothea Bahns, Ludwik Dabrowski, Sergio Doplicher, Klaus Fredenhagen and Giorgio Immirzi for stimulating conversations, and for comments on a preliminary version. Dorothea Bahns drove my attention on reference [13].

\section{References}

[1] Doplicher S., Spacetime and fields, a quantum texture, AIP Conf. Proc. 589 (2001), 204-213, hep-th/0105251.

[2] Doplicher S., Quantum field theory on quantum spacetime, J. Phys. Conf. Ser. 53 (2006), 793-798, hep-th/0608124.

[3] Mead C.A., Possible connection between gravitation and fundamental length, Phys. Rev. 135 (1964), B849B862.

[4] Mead C.A., Wilczek F., Walking the Planck length through history, Phys. Today 54 (2001), 15-15.

[5] Ciafaloni M., Veneziano G., Superstring ñollisions at planckian energies, Phys. Lett. B 197 (1987), 81-88.

[6] Maggiore M., A generalized uncertainty principle in quantum gravity, Phys. Lett. B 304 (1993), 65-69, hep-th/9301067.

[7] Doplicher S., Fredenhagen K., Roberts J.E., The quantum structure of spacetime at the Planck scale and quantum fields, Comm. Math. Phys. 172 (1995), 187-220, hep-th/0303037.

[8] Maggiore M., The algebraic structure of the generalized uncertainty principle, Phys. Lett. B 319 (1993), 83-86, hep-th/9309034.

[9] Snyder H.S., Quantized space-time, Phys. Rev. 71 (1947), 38-41.

[10] Bahns D., Doplicher S., Fredenhagen K., Piacitelli G., Ultraviolet finite quantum field theory on quantum spacetime, Comm. Math. Phys. 237 (2003), 221-241, hep-th/0301100.

[11] Amelino-Camelia G., Doubly-special relativity: facts, myths and some key open issues, Symmetry 2 (2010), 230-271, arXiv:1003.3942.

[12] Dabrowski L., Godlinski M., Piacitelli G., Lorentz covariant k-Minkowski spacetime, Phys. Rev. D 81 (2010), 125024, 8 pages, arXiv:0912.5451.

[13] Cuntz J., Bivariant $k$-theory and the Weyl algebra, K-theory 35 (2005), 93-137, math/0401295.

[14] Rieffel M.A., On the operator algebra for the space-time uncertainty relations, in Operator Algebras and Quantum Field Theory (Rome, 1996), Int. Press, Cambridge, MA, 1997, 374-382, funct-an/9701011.

[15] Estrada R., Gracia-Bondia J.M., Varilly J.C., On asymptotic expansions of twisted products, J. Math. Phys. 30 (1989), 2789-2796.

[16] Aschieri P., Dimitrijević M., Kulish P., Lizzi F., Wess J., Noncommutative spacetimes: symmetries in noncommutative geometry and field theory, Springer, Dordrecht, 2009.

[17] Weyl H., Gruppentheorie und Quantenmechanik, Hirzel, Leipzig, 1928.

[18] von Neumann J., Die Eindeutigkeit der Schrödingerschen Operatoren, Math. Ann. 104 (1931), 570-578.

[19] Piacitelli G., Normal ordering of operator products on quantum spacetime and quantum field theory, PhD Thesis, Universitá di Padova, 2002.

[20] Bahns D., Doplicher S., Fredenhagen K., Piacitelli G., Quantum geometry on quantum spacetime: distance, area and volume operators, arXiv:1005.2130.

[21] Djemai A.E.F., Introduction to Dubois-Violette's noncommutative differential geometry, Internat. J. Theoret. Phys. 34 (1995), 801-887. 
[22] Piacitelli G., Twisted covariance as a non-invariant restriction of the fully covariant DFR model, Comm. Math. Phys. 295 (2010), 701-729, arXiv:0902.0575.

[23] Piacitelli G., Twisted covariance and Weyl quantisation, AIP Conf. Proc. 1196 (2009), 219-224, arXiv:0901.3109.

[24] Chaichian M., Kulish P.P., Nishijima K., Tureanu A., On a Lorentz-invariant interpretation of noncommutative space-time and its implications on noncommutative QFT, Phys. Lett. B 604 (2004), 98-102, hep-th/0408069.

[25] Wess J., Deformed coordinate spaces; derivatives, in Proceedings of the BW2003 Workshop on Mathematical, Theoretical and Phenomenological Challenges Beyond the Standard Model: Perspectives of Balkans Collaboration (2003, Vrnjacka Banja, Serbia), Vrnjacka Banja, 2003, 122-128, hep-th/0408080.

[26] Dabrowski L., Piacitelli G., Poincaré covariant k-Minkowski spacetime, arXiv:1006.5658.

[27] Borowiec A., Pachoł A., $\kappa$-Minkowski spacetimes and DSR algebras: fresh look and old problems, arXiv:1005.4429.

[28] Corinaldesi E., Some aspects of the problem of measurability in quantum electrodynamics, Nuovo Cimento 10 (1953), suppl., 83-100.

[29] Bahns D., Ultraviolet finiteness of the averaged Hamiltonian on the noncommutative Minkowski space, hep-th/0405224.

[30] Bahns D., Doplicher S., Fredenhagen K., Piacitelli G., Field theory on noncommutative spacetimes: quasiplanar Wick products, Phys. Rev. D 71 (2005), 025022, 12 pages, hep-th/0408204.

[31] Bahns D., Doplicher S., Fredenhagen K., Piacitelli G., On the unitarity problem in space-time noncommutative theories, Phys. Lett. B 533 (2002), 178-181, hep-th/0201222.

[32] Gomis J., Mehen T., Space-time noncommutative field theories and unitarity, Nuclear Phys. B 591 (2000), 265-276, hep-th/0005129.

[33] Piacitelli G., Non local theories: new rules for old diagrams, J. High Energy Phys. 2004 (2004), no. 8, 031, 13 pages, hep-th/0403055.

[34] Filk T., Divergencies in a field theory on quantum space, Phys. Lett. B 376 (1996), 53-58.

[35] 't Hooft G., Veltman M.J.G., DIAGRAMMAR, NATO Adv. Study Inst. Ser. B Phys., Vol. 4, Plenum Publishing Corp., New York, 1974, 177-322.

[36] Liao Y., Sibold K., Time-ordered perturbation theory on non-commutative spacetime: basic rules, Eur. Phys. J. C Part. Fields 25 (2002), 469-477, hep-th/0205269.

[37] Liao Y., Sibold K., Time-ordered perturbation theory on noncommutative spacetime. II. Unitarity, Eur. Phys. J. C Part. Fields 25 (2002), 479-486, hep-th/0206011.

[38] Bahns D., Perturbative methods on the noncommutative Minkowski space, PhD Thesis, Universität Hamburg, 2003.

[39] Denk S., Schweda M., Time ordered perturbation theory for non-local interactions: applications to NCQFT, J. High Energy Phys. 2003 (2003), no. 9, 032, 22 pages, hep-th/0306101.

[40] Roepstorff G., Path integral approach to quantum physics. An introduction, Texts and Monographs in Physics, Springer-Verlag, Berlin, 1994.

[41] Bahns D., Schwinger functions in noncommutative quantum field theory, arXiv:0908.4537.

[42] Minwalla S., Van Raamsdonk M., Seiberg N., Noncommutative perturbative dynamics, J. High Energy Phys. 2000 (2000), no. 2, 020, 31 pages, hep-th/9912072. 